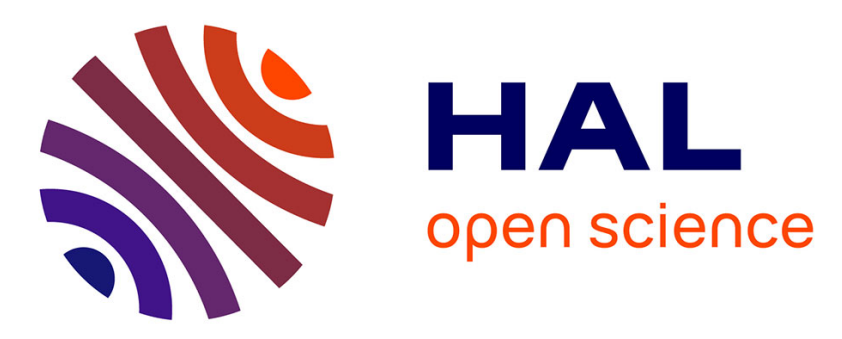

\title{
Experimental and numerical shadowgraph in turbulent Rayleigh-Bénard convection with a rough boundary: investigation of plumes
}

Mebarek Belkadi, Laura Guislain, Anne Sergent, Berengere Podvin, Francesca Chillà, Julien Salort

\section{To cite this version:}

Mebarek Belkadi, Laura Guislain, Anne Sergent, Berengere Podvin, Francesca Chillà, et al.. Experimental and numerical shadowgraph in turbulent Rayleigh-Bénard convection with a rough boundary: investigation of plumes. Journal of Fluid Mechanics, 2020, 895, pp.A7. 10.1017/jfm.2020.296 . hal$02329929 \mathrm{v} 3$

\section{HAL Id: hal-02329929 \\ https://hal.science/hal-02329929v3}

Submitted on 6 Apr 2020

HAL is a multi-disciplinary open access archive for the deposit and dissemination of scientific research documents, whether they are published or not. The documents may come from teaching and research institutions in France or abroad, or from public or private research centers.
L'archive ouverte pluridisciplinaire HAL, est destinée au dépôt et à la diffusion de documents scientifiques de niveau recherche, publiés ou non, émanant des établissements d'enseignement et de recherche français ou étrangers, des laboratoires publics ou privés. 


\title{
Experimental and numerical shadowgraph in turbulent Rayleigh-Bénard convection with a rough boundary: investigation of plumes
}

\author{
Mebarek Belkadi ${ }^{1,2,4}$, Laura Guislain ${ }^{3}$, Anne Sergent ${ }^{1,2}$, Bérengère Podvin ${ }^{1}$, Francesca Chillà ${ }^{3}$, \\ and Julien Salort ${ }^{3}$ \\ ${ }^{1}$ LIMSI, CNRS, Université Paris-Saclay, Campus Universitaire, 91405 Orsay, France \\ ${ }^{2}$ Sorbonne Université, Faculté des Sciences et Ingénierie, UFR d'Ingénierie, 75005 Paris, France \\ ${ }^{3}$ Univ Lyon, ENS de Lyon, Univ Claude Bernard, CNRS, Laboratoire de Physique, Lyon, France \\ ${ }^{4}$ Laboratory of Turbomachinery, Ecole Militaire Polytechnique, Bordj El Bahri, 16111, Algiers, \\ Algeria
}

Monday, March 6th, 2020

\begin{abstract}
We show that, in the case of turbulent Rayleigh-Bénard convection, shadowgraph can be used to have quantitative results on the plumes statistics and their velocity. For this purpose, we compare the experimental shadowgraph of a Rayleigh-Bénard cell with the synthetic shadowgraph obtained by calculating the integrated 2D Laplacian of the temperature field from a numerical simulation very similar to the experiment.

We use image processing tools to enhance the quality of the shadowgraph image, and obtain quantitative statistics for the thermal plumes, such as plume density, and plume velocity distribution. To highlight the efficiency of this new process of plume counting, we use, both in the experiment and in the numerical simulation, a turbulent Rayleigh-Bénard convection cell with a rough bottom surface, and a smooth top surface, where the statistics of the plumes can be influenced (or not) by the roughness. In addition, the distribution of velocity obtained from processing the synthetic shadowgraph images of the DNS or the experimental shadowgraph images, are compared to the velocity fluid at mid plane of the DNS or PIV measurement in the experiment, respectively. It will be shown that the mean velocity profile measured using the advection of the plumes is different from the average eulerian velocity profile.
\end{abstract}

\section{Introduction}

The Rayleigh-Bénard cell, which consists in a fluid layer heated from below and cooled from above, is a useful model system to investigate turbulent thermal convection. It is very widely used, for both experimental and numerical studies (Ahlers et al., 2009; Chillà \& Schumacher, 2012). The main non dimensional control parameter is the Rayleigh number, $R a$,

$$
R a=\frac{g \alpha \Delta T h^{3}}{\nu \kappa}
$$

which compares the buoyancy forcing and the diffusive terms, where $g$ is the acceleration of gravity, $\alpha$ the thermal expansion coefficient, $\Delta T$ the temperature difference, $h$ the cell height, $\nu$ the kinetic viscosity, and $\kappa$ the thermal diffusivity. Turbulent thermal convection is obtained when $R a>10^{7}$. The other control parameters are the Prandtl number, $P r$, and the cell aspect ratios, $\Gamma_{x z}$ and $\Gamma_{y z}$,

$$
\operatorname{Pr}=\frac{\nu}{\kappa} \text { and } \Gamma_{x z}=\ell / h \text { and } \Gamma_{y z}=D / h,
$$

where $\ell$ is the cell width and $D$ the cell depth.

The flow can be characterized by global quantities, such as the global heat transfer efficiency, which can be quantified by the Nusselt number, $N u$,

$$
N u=\frac{\dot{q} h}{\lambda \Delta T},
$$


where $\dot{q}$ is the thermal flux, and $\lambda$ is the thermal conductivity. When heat is transported by convection, the Nusselt number is larger than unity. There have been a lot of efforts to find the relation between the Nusselt number and the control parameters (Castaing et al., 1989; Shraiman \& Siggia, 1990; Grossmann \& Lohse, 2000, 2011), in particular in the high Rayleigh number limit where the flow is highly turbulent (see Ahlers et al. (2009); Chillà \& Schumacher (2012) for reviews of experiments, numerical simulations and scaling theories).

Another approach is to investigate quantities, such as velocity and temperature. Experimental investigation of the velocity field often requires optical access to the cell, and the seeding of particles, (Qiu \& Tong, 2001; Xia et al., 2003; Zhou et al., 2011; Ni et al., 2012; Liot et al., 2016a, 2017). However, optical access is not always possible, in particular in the cryogenic set ups (Chavanne et al., 1997; Niemela et al., 2000; Urban et al., 2014), or in the Göttingen sulphur hexafluoride experiment (He et al., 2012a), and most traditional local velocity sensors — hot wires, Pitot tubes - cannot operate in the velocity range of thermal convection. The alternative method which has been used to infer velocity estimates in these system is based on the correlation of two or more thermometers (Wu, 1991; Chavanne et al., 2001; Sun \& Xia, 2005; He et al., 2015; Musilová et al., 2017).

The experimental investigation of the temperature field is a difficult matter, because of the low amplitude of temperature fluctuations in the bulk. Direct measurements can be achieved with thermochromic liquid crystals (TLC) (Du \& Tong, 2001; Stasiek \& Kowalewski, 2002; Zhou et al., 2007a; Zhou \& Xia, 2010; Tummers \& Steunebrink, 2019), or temperature sensitive fluorescent dye (Sakakibara \& Adrian, 2004). Though quite successful, the drawbacks of these methods are the technical difficulty to increase the sensitivity and the response time, enough for the very large Rayleigh number limit. The simple alternative is the imaging of the optical index gradient using either shadowgraph (de Bruyn et al., 1996; Jenkins, 1988; Trainoff \& Cannell, 2002; Xi et al., 2004; Hattori et al., 2013; Wang et al., 2018), or schlieren (Ciliberto et al., 1985; Chillà et al., 1993; Dalziel et al., 2000; Salort et al., 2014; Raffel, 2015; Taberlet et al., 2018). The advantage is that these methods are straightforward to implement, non-invasive, and are fairly sensitive. However, the flow pattern is averaged over the depth of the cell.

It can be mentionned also, that there have been studies which infer plume statistics from one local temperature sensor. For example, Gauthier et al. (2009) evidenced a transition in the temperature fluctuation statistics in the ultimate regime of convection, Zhou et al. (2016) studied the width and amplitude statistics of plume with Rayleigh numbers ranging from $2 \times 10^{8}$ to $1.85 \times 10^{10}$. There are also many investigations of temperature profiles (Tilgner et al., 1993; du Puits et al., 2007; Ahlers et al., 2012; Wei \& Ahlers, 2014; Xie et al., 2019).

The first goal of this paper is to discuss some quantitative information that can be inferred from an experimental shadowgraph image in the Rayleigh-Bénard system. The recent study of Wang et al. (2018) show that adequate post-processing effectively allows to derive useful quantities (in their case, statistical properties of eruption events of thermal plumes in a vertical thin disk convection experiment). To that end, experimental shadowgraph images are compared with direct numerical simulations (DNS) of a Rayleigh-Bénard cell, from which a synthetic shadowgraph image can be computed from the temperature field, under the assumption that geometrical optics holds (Trainoff \& Cannell, 2002). The results are compared against the fluid velocity fields in the case of the computer simulations, and against Particle Image Velocimetry (PIV) measurements in similar conditions in the case of the experimental data.

One important open question in turbulent thermal convection is to understand and accurately model the flow and heat transfer efficiency in the highly turbulent limit. Kraichnan (1962) predicted that turbulent boundary layers would increase the scaling law for the Nusselt number to the asymptotic regime

$$
N u \sim R a^{1 / 2}[\log R a]^{-3 / 2} .
$$

The search for this asymptotic regime, and for evidence of turbulent boundary layers in turbulent Rayleigh-Bénard convection, has been a long term effort from many groups and is still a very active topic (Chavanne et al., 1997; Niemela et al., 2000; Funfschilling et al., 2009; Chillà et al., 2004; Roche et al., 2010; Grossmann \& Lohse, 2011; He et al., 2012b; Ahlers et al., 2017; Zhu et al., 2018a; Bouillaut et al., 2019; Urban et al., 2019). However, the range of Rayleigh numbers that must be achieved to enter this regime is very high, and not easily accessible to experiments or numerical simulations. Experiments are possible only with non conventional fluids such as cryogenic helium and pressurized sulphur hexafluoride. Additionally, the threshold seems to highly depend on small details, which led to apparent contradictions between experimental results.

An alternative approach is to attempt to lower the threshold Reynolds number with a surface roughness, and seek evidence of a transition to turbulent boundary layers at a lower and more accessible Rayleigh number. While this approach has proved at least partially successful in the sense that roughness surfaces do trigger enhanced heattransfer, and higher scaling exponent (Salort et al., 2014; Liot et al., 2016b; Toppaladoddi et al., 2017; Zhu et al., 2017; Xie \& Xia, 2017; Zhu et al., 2018b), the detailed mechanisms are still open for debate, and the dynamics is very rich. In particular, Zhu et al. (2017) and MacDonald et al. (2019) claim that the roughness-facilitated 
local $1 / 2$ scaling is different in nature from the high-Rayleigh number asymptotically $1 / 2$ scaling (ultimate regime). In contrast, Toppaladoddi et al. (2017) claim that roughness facilitates the attainment of the ultimate regime. The present paper will not settle this debate, but will contribute new experimental and numerical data into the discussion. Thermal convection over rough surfaces is also an important question for real natural and industrial applications, since most natural surfaces are not hydrodynamically smooth. That is why the analysis of the heat transfer efficiency in thermal convection over rough surfaces has triggered both experimental (Du \& Tong, 1998; Ciliberto \& Laroche, 1999; García et al., 2012; Wei et al., 2014; Xie \& Xia, 2017; Rusaouen et al., 2018; Tummers \& Steunebrink, 2019), theoretical (Shishkina \& Wagner, 2011; Goluskin \& Doering, 2016), and numerical efforts (Wagner \& Shishkina, 2015; Toppaladoddi et al., 2017; Zhu et al., 2017; Zhang et al., 2018; Zhu et al., 2019).

The most consensual result is that a heat transfer enhancement is triggered when the thermal boundary layer thickness matches the height of the roughness size. The nature of the enhanced regime may however differ: some observe an increase of the scaling exponent (Roche et al., 2001; Qiu et al., 2005; Tisserand et al., 2011), and some observe only an increase of the prefactor (Du \& Tong, 1998). There is also a competition between an enhancement of plume emission by the rough surface, as directly evidenced by Du \& Tong (2000) with thermochromic liquid crystals, and an enhancement due to higher turbulence intensity (Salort et al., 2014).

Recent works suggest that three regimes can be identified (Xie \& Xia, 2017; Zhu et al., 2017; Rusaouen et al., 2018; Belkadi, 2019; Tummers \& Steunebrink, 2019): Regime I when the surface is hydrodynamically smooth, Regime II when the plate becomes hydrodynamically rough and triggers a change in the scaling law exponent, and Regime III when the heat transfer enhancement saturates due to the viscous boundary layer cross-over. These regimes allow to understand the apparent discrepancies previously reported.

In this work, the experiment and the numerical simulation are not in the same regime. The experiment is operated in Regime II, and the numerical simulation in Regime III. Indeed, we use the numerical simulation, where all the fields are known, to study the processing tools. The results can be compared to experimental measurements, because the processing tools are the same to study all regimes. The limits of the plume detection method does not depend on the regime, and comparison remains relevant.

Therefore, we present the experiment and the numerical simulation side by side, even though the statistical features of the plumes in both case are not expected to be similar. Once the detection method is established, the fact that the experiment and the numerics lie respectively in Regime II and Regime III, allows to highlight particular features of each regime.

The second goal of this paper is thus to use the quantitative information on the thermal plumes, provided by the analysis of the shadowgraph image, to compare the statistics of the warm plumes emitted by the rough surface, with those of the cold plumes emitted by the smooth surface, in both cases. We previously showed that the smooth half-cell behaves, at first order, as a smooth convection cell, at least in the range of Rayleigh numbers that we investigate, where roughness triggers more intense turbulence fluctuations. And the rough half-cell essentially behaves as a rough convection cell. These allow to directly compare the dynamics of smooth and rough plates, within the same system.

The paper is structured with the experimental and numerical results side by side. The experimental and numerical configurations are described in Section 2. The methods to obtain experimental and numerical shadowgraph images are described in Section 3. The statistics of plume velocity and plume density, based on the analysis of spatio-temporal diagrams, is discussed in Section 4.

\section{Rayleigh-Bénard convection cells}

\subsection{Experimental configuration}

The experimental convection cell is $41.5 \mathrm{~cm} \times 10.5 \mathrm{~cm} \times 41.5 \mathrm{~cm}$ rectangular cell, with aspect ratios $\Gamma_{x z}=1$ and $\Gamma_{y z}=0.253$. The details of this cell have been published in our previous works (Salort et al., 2014; Liot et al., 2017). The only difference is that the walls are now made of glass, instead of PMMA, to avoid spurious optical index gradients in the walls. Because the heat conductivity of glass is larger than PMMA, this means that we have to work with a bulk temperature close to the temperature of the room, to avoid heat flux across the walls. Because the cell cannot be fully insulated to preserve optical access, only sufficiently high heating power can be used, for the heat losses to remain negligible. The experimental conditions are summarized in table 1.

We recall the main characteristics. The bottom plate is rough, made of aluminium alloy (5083) anodized in black. The roughness consists in periodic parallelepipeds roughness elements $2 \mathrm{~mm} \times 5 \mathrm{~mm}$, machined directly into the plate. The top plate is $4 \mathrm{~cm}$-thick smooth copper plate coated with a thin layer of nickel. Plate temperature are measured with PT-100 temperature sensors. The bottom plate is heated with kapton heaters glued at the bottom of 


$\begin{array}{rlll} & \text { Shadowgraph } & \text { PIV } & \text { DNS } \\ \text { Walls } & \text { Glass } & \text { PMMA } & \text { Adiabatic BC } \\ \text { Heating power } & 400 \mathrm{~W} & 400 \mathrm{~W} & \\ \text { Cell height } h & 41.5 \mathrm{~cm} & 41.5 \mathrm{~cm} & 40 \mathrm{~cm} \\ \text { Cell width and depth } \ell \times D & 41.5 \mathrm{~cm} \times 10.5 \mathrm{~cm} & 41.5 \mathrm{~cm} \times 10.5 \mathrm{~cm} & 40 \mathrm{~cm} \times 20 \mathrm{~cm} \\ \text { Roughness height } H_{p} & 2 \mathrm{~mm} & 2 \mathrm{~mm} & 1.2 \mathrm{~cm} \\ \text { Roughness width and depth } \ell_{p} \times D_{p} & 5 \mathrm{~mm} \times 5 \mathrm{~mm} & 5 \mathrm{~mm} \times 5 \mathrm{~mm} & 3 \mathrm{~cm} \times 3 \mathrm{~cm} \\ T_{\text {mean }} & 39.4^{\circ} \mathrm{C} & 42.7^{\circ} \mathrm{C} & 40^{\circ} \mathrm{C} \\ \Delta T & 21.3 \mathrm{~K} & 22.1 \mathrm{~K} & 0.85 \mathrm{~K} \\ R a & 5.6 \times 10^{10} & 6.2 \times 10^{10} & 2 \times 10^{9} \\ N u & 298.2 & 287.2 & 107.0 \pm 0.54 \% \\ P r & 4.4 & 4.1 & 4.38\end{array}$

Table 1: Experimental and numerical configurations of the Rayleigh-Bénard cells. BC: boundary conditions. The DNS Nusselt number is given as the mean and its deviation of several estimations as proposed by Shishkina et al. (2010).

the plate, and powered with a constant voltage. The top plate is cooled with a circulation of a mixture of water and ethylene glycol, flowing into a tight meander machined at the top of the copper plate, and temperature regulated by a Lauda RP845 chiller.

\subsection{Numerical configuration}

The numerical configuration is detailed in Belkadi (2019). We consider a physical configuration close to the experimental one: a $40 \mathrm{~cm}$ high Rayleigh-Bénard cell of a square vertical cross-section, filled with water at the mean temperature $\left(T_{\text {mean }}\right)$, with a rough bottom plate, and a smooth top plate. The roughness consists in regularly spaced parallelepiped roughness elements. The main characteristics of the physical problem are given in table 1 . In this paper, the Rayleigh number is set to $R a=2 \times 10^{9}$.

The fluid flow is governed by the Boussinesq equations, where the gravity vector $\mathbf{g}$ is aligned with the $-\mathbf{e}_{z}$ direction. A no-slip condition for the velocity field is applied on the walls. The temperature difference $\Delta T$ is imposed on the top and bottom walls including the roughness element surfaces by applying isothermal boundary conditions $\left(T_{\text {top }}=T_{\text {mean }}-\Delta T / 2\right.$ and $\left.T_{\text {bot }}=T_{\text {mean }}+\Delta T / 2\right)$, while adiabaticity $\frac{\partial T}{\partial \mathbf{n}}=0$ is satisfied on side walls.

The Boussinesq solver called SUNFLUIDH uses a finite volume discretization on staggered grids. The time marching is treated by a semi-implicit scheme combined with a prediction-correction scheme for the velocity-pressure coupling, with a global second-order accuracy. The pressure problem is solved by a multi-grid method. Roughness elements are modelled through a loop truncation technique. A domain decomposition method using MPI is implemented for parallel computation.

The simulation has been performed on a non-uniform grid with $768 \times 384 \times 768$ cells in $\left(\mathbf{e}_{x}, \mathbf{e}_{y}, \mathbf{e}_{z}\right)$ directions respectively. The mesh has been particularly refined between roughness elements, and the cell size is everywhere finer than the Kolmogorov scale. The time step, equal to 0.011925 seconds, meets the CFL criterion. The spatial resolution has been checked by evaluating the numerical convergence of time-averaged Nusselt numbers obtained by different methods (space averages on the horizontal planes or volume averages based on the dissipation rates) as proposed by Shishkina et al. (2010). Statistics have been made over more than one hours and 20 minutes (physical time) to obtain these values. They converge with a deviation of less than $1 \%$ of the average Nusselt number, as specified in table 1.

\section{Shadowgraph images}

\subsection{Experimental setup}

There are several possible shadowgraph set ups (Settles, 2001). The classical, and most used set up requires parallel incident light. This is obtained with either a concave mirror or a lens. However, for field of views larger than $15 \mathrm{~cm}$, this is impractical, or would require using parabolic telescope mirror. The alternative, that we used in this work, is to implement direct shadowgraphy in diverging light with a small, or punctual, bright source of light. This is the method originally used by Marat (1780). 


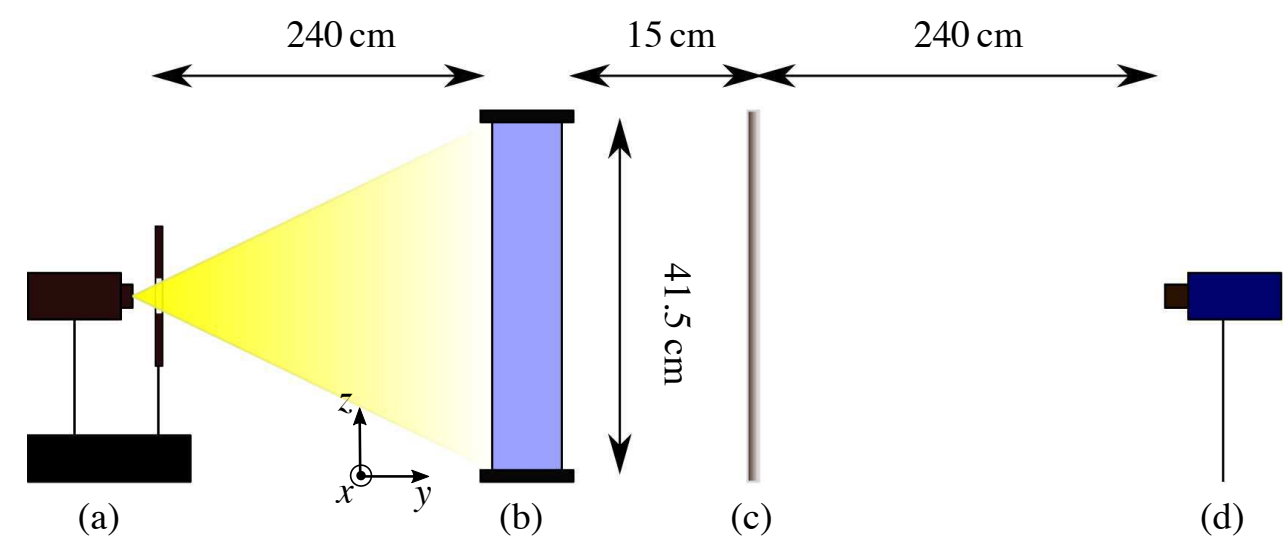

Figure 1: Optical set-up for the shadowgraph experiment. (a) Quartz Iodine Lamp, heat absorbing filter, and diaphragm, (b) Rayleigh-Bénard convection cell, (c) ground glass diffuser, (d) PCO 1600 camera.

In this work, the convection cell is illuminated by a punctual source of white light, as shown in figure 1 . The cell depth is four times smaller than the width and height, so that the flow is quasi bidimensional and the integration along the depth does not significantly blur the convection patterns.

The shadowgraph pattern is captured on a projection screen, and recorded with a PCO 1600 digital camera. The resolution is 1600 pixels $\times 1200$ pixels and the frame rate is $10 \mathrm{fps}$, corresponding to 800 images per turnover, where the turnover time $T_{\text {turnover }}$ is

$$
T_{\text {turnover }}=\frac{4 h}{v_{\text {wind }}},
$$

and $v_{\text {wind }} \approx 2 \mathrm{~cm} / \mathrm{s}$. 20 series of 2400 images were recorded, amounting to 80 minutes which is to 60 turnover times. The recorded image, $I(x, z)$, is determined by the second derivative of the refractive index,

$$
\frac{I(x, z)}{I_{0}}=\frac{\gamma(\eta-\gamma)}{\eta} \int_{0}^{D}\left(\frac{\partial^{2}}{\partial x^{2}}+\frac{\partial^{2}}{\partial z^{2}}\right)(\ln n) \mathrm{d} y
$$

where $I_{0}$ is the intensity of the incident light, $\gamma=15 \mathrm{~cm}$ is the distance between the cell and the projection screen, and $\eta=265 \mathrm{~cm}$ is the distance between the light source and the projection screen. Since in this setup, $\eta / \gamma \gg 1$, the sensitivity function $\gamma(\eta-\gamma) / \eta$ is not very large, and nearly proportional to $\gamma$, and the magnification factor $m$

$$
m=\frac{\eta}{\eta-\gamma},
$$

is close to unity. This is close to the case of parallel light, and is sufficient to image plumes in Rayleigh-Bénard convection.

In practice, in the case of thermal convection, the variation of optical index is caused by variations of temperature, which are small in the bulk. Eq. 6 can thus be written in a simpler form,

$$
\frac{I(x, z)}{I_{0}} \propto \gamma \int_{0}^{D}\left(\frac{\partial^{2} T}{\partial x^{2}}+\frac{\partial^{2} T}{\partial z^{2}}\right) \mathrm{d} y .
$$

A snapshot of the experimental shadowgraph is shown in figure 2-a. The raw image is numerically enhanced by dividing the field by the reference image $I_{0}(x, z)$. This reference image can be obtained with the fluid at rest, or by averaging the recording in time. Indeed, the plume pattern is lighter and darker, and averages to zero. We found no practical difference between the two estimates of $I_{0}(x, z)$, and found more practical to use the time average. As shown in the figure, the variations of light intensity induced by the thermal plumes is $\pm 20 \%$.

\subsection{Synthetic shadowgraph in numerical simulations}

A numerical shadowgraph can be derived from the temperature field using Eq. 8, at regular time steps $0.5366 \mathrm{~s}$, and produce a sequence of synthetic images, similar to those obtained in the experiments (see figure 2-b). To that end, 5524 integrated 2D-Laplacian fields were saved, spanning over 49 minutes in dimensional time, with a numerical 
(a)

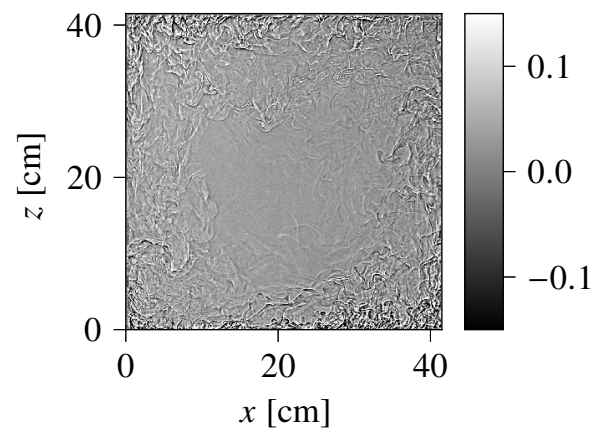

(c)

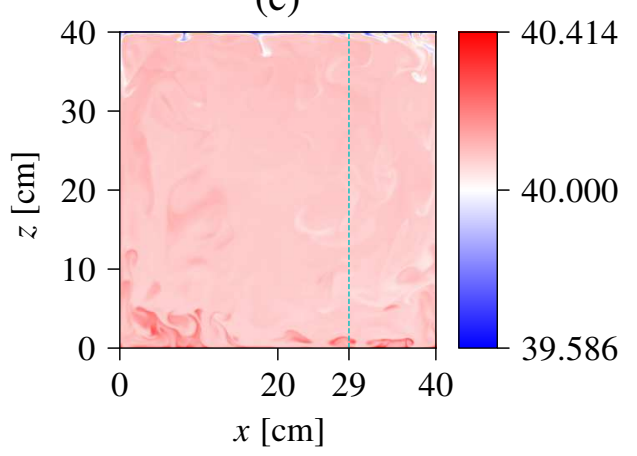

(b)

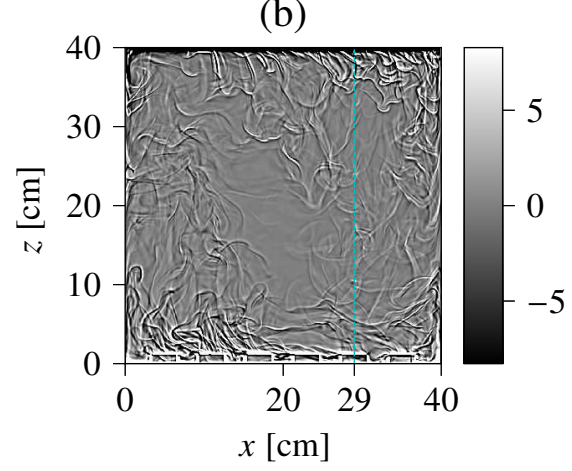

(d)

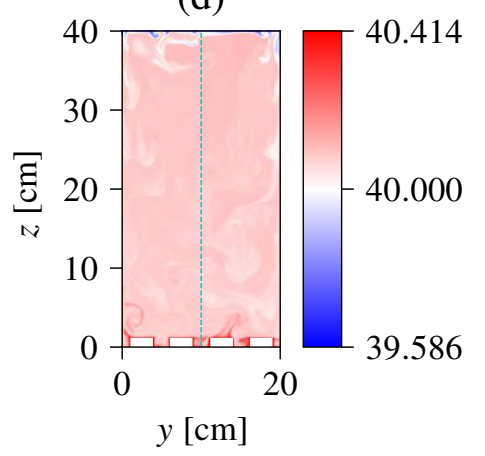

Figure 2: (a) Experimental full field shadowgraph image at $R a=5.6 \times 10^{10}$. The colour bar indicates the relative light intensity, $I(x, z) / I_{0}(x, z)-1.0$, where $I_{0}(x, z)$ is the time-averaged image of the cell (see text). (b) Synthetic full field shadowgraph image from the numerical simulation at $R a=2 \times 10^{9}$, computed from the full 3D temperature field using Eq. 8. (c) Snapshot of the mid-plane $(y=10 \mathrm{~cm})$ temperature of the numerical simulation at the same time as the shadowgraph. Only the fluid trapped between the roughness elements is visible at this particular position (see the dashed line in figure 2(d)). (d) Snapshot of the transverse $(x=29 \mathrm{~cm})$ temperature. The blue dashed lines in (b), (c) and (d) show the intersection of the planes. 
(a)

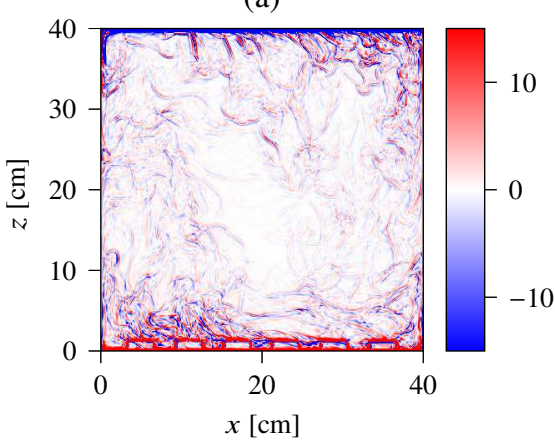

(c)

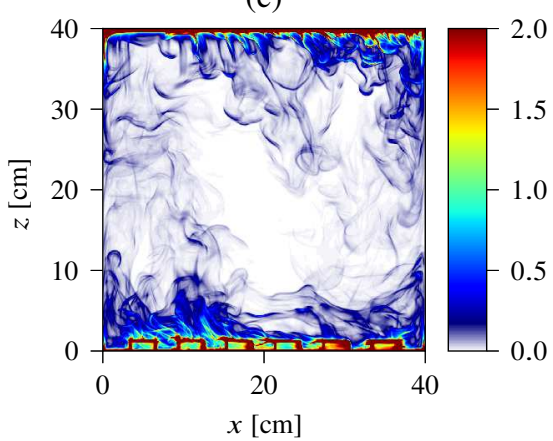

(b)

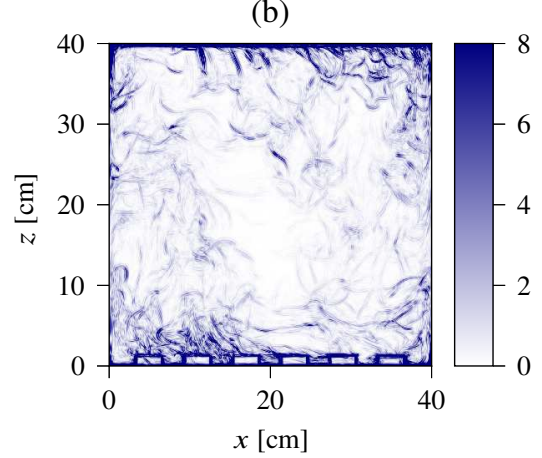

(d)

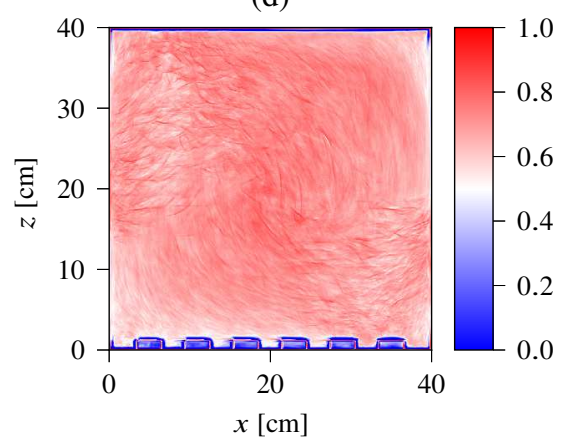

Figure 3: Snapshot of the numerical fields at $R a=2 \times 10^{9}$. (a) Numerical shadowgraph computed with Eq. 8 . (b) Local variance of the shadowgraph image. (c) Local $2 \mathrm{D}$ thermal dissipation rate, averaged over $y,\left\langle\epsilon_{\theta, 2 D}\right\rangle_{y}=$ $\left\langle\kappa\left(\left(\partial_{x} T\right)^{2}+\left(\partial_{z} T\right)^{2}\right)\right\rangle_{y}$, normalised by the global thermal dissipation rate, $\left\langle\epsilon_{\theta}\right\rangle_{x, y, z, t}=\kappa\left(\Delta T^{2} / h^{2}\right) N u$. (d) Timeaveraged cross-correlation between the local variance of the shadowgraph image and the local 2D thermal dissipation rate averaged over $y$ (average value is $63.8 \%$ ).

frame rate of $1.86 \mathrm{fps}$. The mean wind is $0.4 \mathrm{~cm} / \mathrm{s}$, so the numerical frame rate corresponds to 745 images per turnover, and a total recording time of 7.4 turnovers. The time resolution is therefore of similar order of magnitude as the experimental recording frame rate, but with a shorter duration in terms of turnover times. However, the Rayleigh number is also smaller, so fewer turnover times are enough to reach statistical convergence. This allows to use the same type of image, and similar processing tools, both for the experiment and the numerics.

The comparison with the experimental shadowgraph, shown in figure 2-a, evidence more plumes, and smaller plumes in the experiment. This is due to the difference in Rayleigh numbers. The numerical shadowgraph of the present work can also be compared to experimental shadowgraphs obtained in water at lower Rayleigh numbers (Wang et al., 2018; Tummers \& Steunebrink, 2019), and higher Rayleigh numbers (Xie \& Xia, 2017).

In this paper, we focus on the analysis of the shadowgraph itself, and use the numerical simulations to validate the interpretation of the field. To that aim, it is not necessary to have experiments and numerical simulations in the same range of Rayleigh numbers. The role of Rayleigh number, and the various regimes in rough cells, is beyond the scope of the present work, but will benefit from the tools described here, in particular in situations where shadowgraph may be the only available investigation tool.

A snapshot of the mid-plane temperature field is shown in figure 2-c. The mean temperature value is larger than $40^{\circ} \mathrm{C}$, because of the enhanced heat transport of the rough surface at the bottom. Only a few plumes can be identified on the temperature snapshot, both on the top and bottom surfaces, while much more plumes can be seen on the numerical shadowgraph. This is due to the integration along $y$ in the latter, which adds up contributions of plumes at several $y$ position, as well as the contribution of their 3D structure. The far lower number of visible plume on the mid-plane temperature, versus integrated fields such as shadowgraph, can also be observed experimentally, e.g. with laser-induced fluorescence (Sakakibara \& Adrian, 2004), or thermochromic liquid crystals (Du \& Tong, 2001), but there are few direct comparisons.

Figure 3 compares the synthetic shadowgraph image, computed from Eq. 8, i.e. the $y$-averaged 2D Laplacian of 

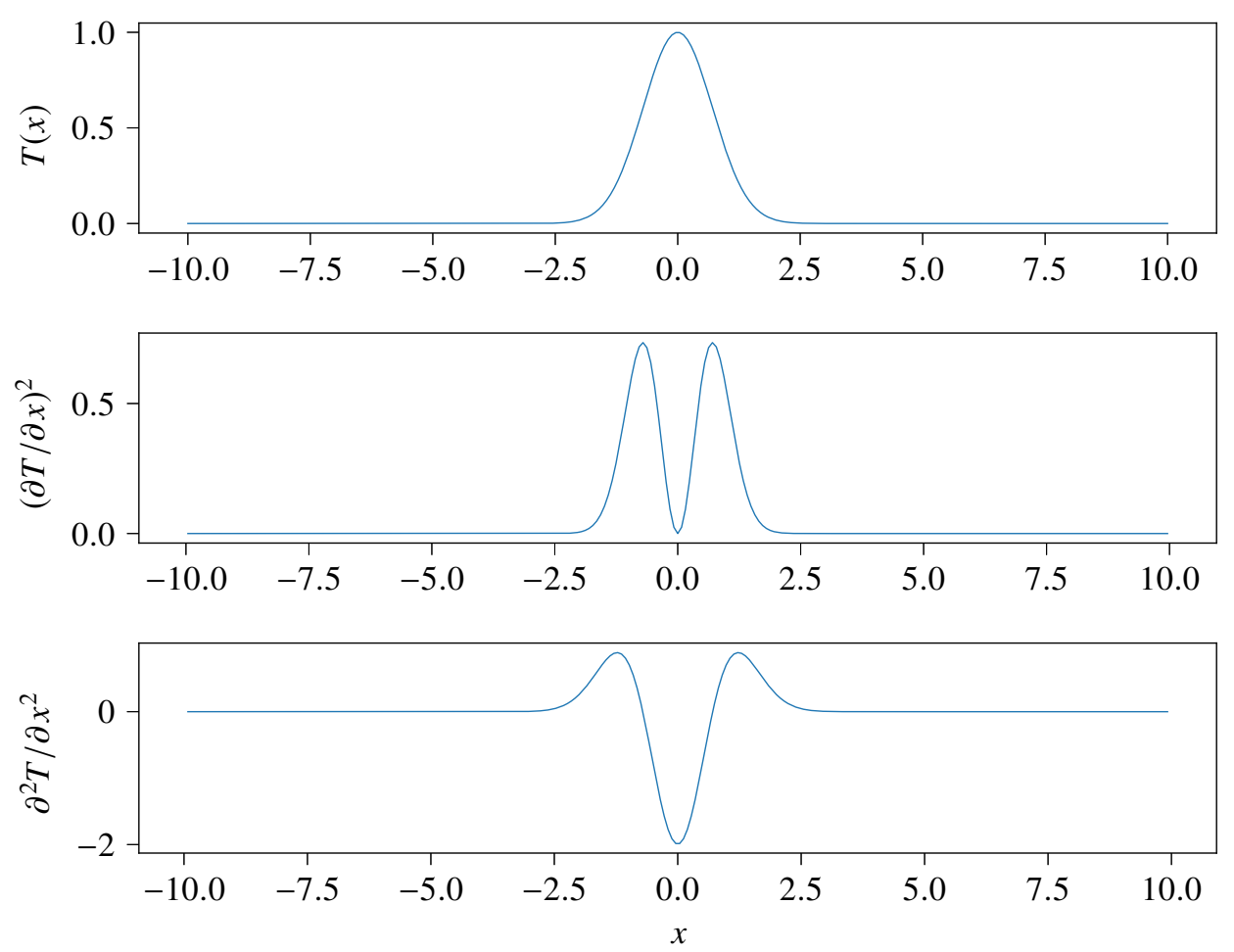

Figure 4: Simple sketch of patch of hot fluid. (a) Temperature profile. (b) Thermal dissipation profile. (c) Shadowgraph profile.

temperature, and the $y$-averaged $2 \mathrm{D}$ thermal dissipation rate, $\left\langle\epsilon_{\theta, 2 D}\right\rangle_{y}$, which is given by

$$
\left\langle\epsilon_{\theta, 2 D}\right\rangle_{y}(x, z)=\kappa\left\langle\left(\frac{\partial T}{\partial x}\right)^{2}+\left(\frac{\partial T}{\partial z}\right)^{2}\right\rangle_{y}
$$

There is no straightforward mathematical relation between $I(x, z)$ and $\left\langle\epsilon_{\theta, 2 D}\right\rangle_{y}$ in the general case. However, figure 4 sketches the case of a warm fluid parcel over a mostly isothermal background. It is represented by a Gaussian profile for simplicity, but similar result would be obtained for other bell-shaped curve. The dissipation rate,

$$
\epsilon_{\theta} \sim(\partial T / \partial x)^{2},
$$

and the Laplacian,

$$
\partial^{2} T / \partial x^{2}
$$

have similar shape with two maxima located roughly at the warm parcel mid-height.

In the case of the Laplacian, the maxima are positive, and the minimum is negative. This can be observed in both the experimental and numerical shadowgraph image, where a plume pattern consists in darker and lighter boundaries (see figure 2 and 3). The thermal dissipation rate is always positive, and more closely resembles the shadowgraph local variance (discussed in the next section). The qualitative conclusion is that the structures evidenced in shadowgraph images correspond to both areas of high thermal dissipation rate marking out the plume, which could also be used as a criterion to detect thermal plumes (Belkadi, 2019).

To estimate the similarity of the local variance of the shadowgraph image (figure 3 -b) and the $y$-averaged thermal dissipation, $\left\langle\epsilon_{\theta, 2 D}\right\rangle_{y}$ (figure 3-c), one can compute the time-averaged cross-correlation, $C(x, z)$,

$$
C(x, z)=\frac{\langle S(x, z, t) D(x, z, t)\rangle_{t}-\langle S(x, z, t)\rangle\langle D(x, z, t)\rangle_{t}}{\left(\left\langle S(x, z, t)^{2}\right\rangle_{t}-\langle S(x, z, t)\rangle_{t}^{2}\right)^{1 / 2} \times\left(\left\langle D(x, z, t)^{2}\right\rangle_{t}-\langle D(x, z, t)\rangle_{t}^{2}\right)^{1 / 2}},
$$


where $S(x, z, t)$ is the local variance of the shadowgraph, and $D(x, z, t)$ is the $y$-averaged thermal dissipation. As shown in figure $3-\mathrm{d}$, the cross-correlation is higher than $60 \%$ almost everywhere in the cell, and it averages to $63.8 \%$. This shows that the two fields are indeed correlated.

\section{Spatio-temporal diagrams}

\subsection{Plume detection technique}

One common way to find advected structures in a sequence of images is to build a spatio-temporal diagram, $I\left(x, z_{0}, t\right)$, by choosing a vertical position $z_{0}$, or $I\left(x_{0}, z, t\right)$, by choosing a horizontal position $x_{0}$, and plotting the image intensity as a function of $x$ and $t$, resp. $z$ and $t$. As shown in figure 5 , advected coherent structures yield inclined lines. Similarly to other such analysis in Rayleigh-Bénard convection (Chillà et al., 1993; Taberlet et al., 2018), these lines are a clear evidence of the mean wind in opposite direction near each plate, and no clear advection at the centre.

By measuring the slope of those segments, one can get estimates for the average horizontal velocity (resp. the vertical velocity), and a density of plumes. This method is very common in the field of geophysical fluid dynamics, where it is used to find propagating wave fronts, and is sometimes referred to as Hovmöller diagram (Lovegrove et al., 2000; Flór et al., 2011).

In the remainder of the paper, we use the terms plume velocity to refer to velocities obtained from the analysis of plume advection (shadowgraph spatio-temporal diagrams, or "time-of-flight" correlation time from thermometers), and fluid velocity to refer to either the numerical simulation velocity field, or experimental Particle Image Velocimetry (PIV) measurements.

To obtain a spatio-temporal diagram that can be further successfully post-processed, the contrast of the image must first be enhanced, so that plumes are more visible. The ultimate contrast would consist in texture segmentation, where pixels that belong to a plume are separated from pixels that belong to the bulk. One possible estimate for this type of task is based on the local variance and the local regularity (Pascal et al., 2018).

A local variance image is obtained from a standard deviation filtering operation, which consists in computing a new image where each pixel is the standard deviation of the neighbourhood around the corresponding pixel in the original image. High values in this new image correspond to areas where there are large changes in the original image intensity. Low values correspond to areas where the original image intensity is more homogeneous. Hence, boundaries of plumes where there are sharp temperature gradients will produce high local variance values.

Figure 6 -a and c shows the local variance of the shadowgraph image, computed on neighbourhoods of $3 \times 3$ pixels, both for the experimental shadowgraph and for the numerical shadowgraph. The contrast is indeed well enhanced.

In the case of the numerical shadowgraph, the resolution is enough to observe the spatial oscillation pattern of each plume. Larger values of neighbourhood would not remove these oscillations, but led to blurred images. As previously discussed, they are the result of the double derivative of the spatial temperature profile of the plume (see sketch in figure 4), and should also be visible on experimental shadowgraph. However, the plumes in the experiments are much smaller, compared to the resolution of the camera, to fully resolve the structure within each plume, and the oscillation patterns is no longer visible on the local variance image.

A threshold applied on the local variance yields a segmented picture, where pixels belonging to a plume are separated from those belonging to the bulk. However, in the case of Rayleigh-Bénard convection, the plume density is too high for such a binary decomposition, as can be seen in figure 6-b and d. Therefore, we use the local variance only to enhance the contrast. The spatio-temporal diagrams computed from the local variance images are shown in figure 7 for the case of a horizontal line near the top of the cell, and in figure 8 for the case of a vertical line at $x_{0}=8 \mathrm{~cm}$.

The experimental and numerical spatio-temporal diagrams are very similar, and also closely resemble the diagrams obtained by Chillà et al. (1993) from the temperature gradient with a sweeping laser method at a lower Rayleigh number $\left(R a=10^{8}\right)$. All feature inclined lines which are a signature of the advection of plumes.

In the present work, the contrast of the inclined lines is good enough to proceed to the detection of line segments. We use the algorithm of Grompone von Gioi et al. (2012), implemented in the free OpenCV library (Bradski, 2000). The detected segments in the case of a spatio-temporal diagram built from a horizontal line at $z=z_{0}$, are shown in figure 7-b and d,restricted to segments at the centre of the cell, $\xi<x<\ell-\xi$, and with durations $\tau$ of several time steps. For both the experimental and numerical line segments, we choose $\xi=\ell / 4$, to keep only the contribution of the nearly horizontal wind, and remove the corner flows and the jets. The duration is $\tau=0.4 \mathrm{~s}$ for the experimental data, and $\tau=2.3 \mathrm{~s}$ for the numerical data. The difference comes from the typical velocity that is much lower in the numerical setup, due to the lower Rayleigh number. 
(a)

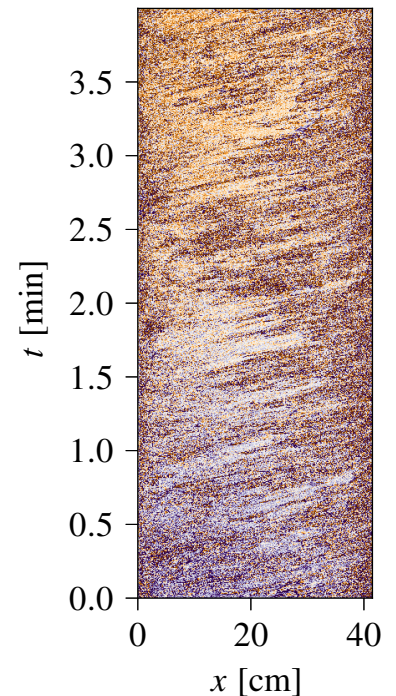

(d)

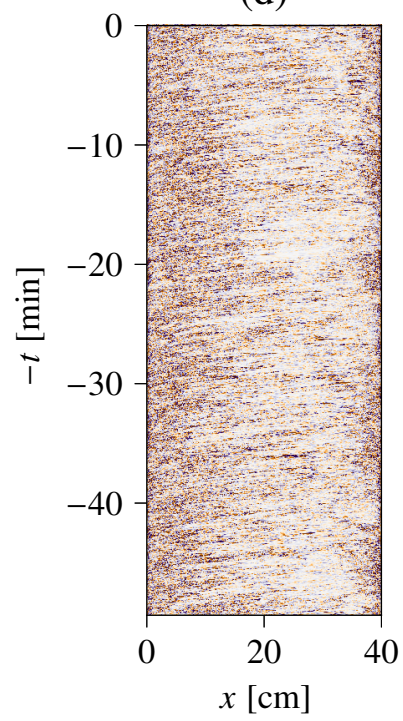

(b)

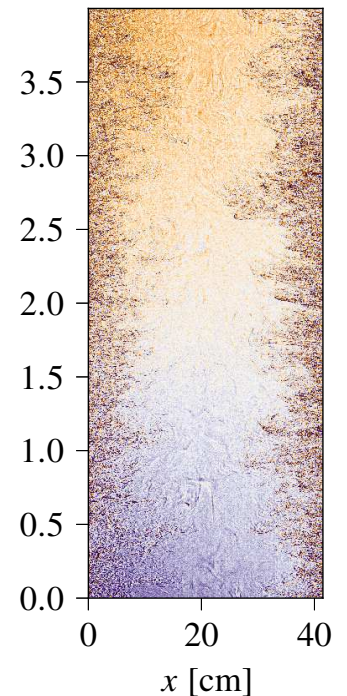

(e)

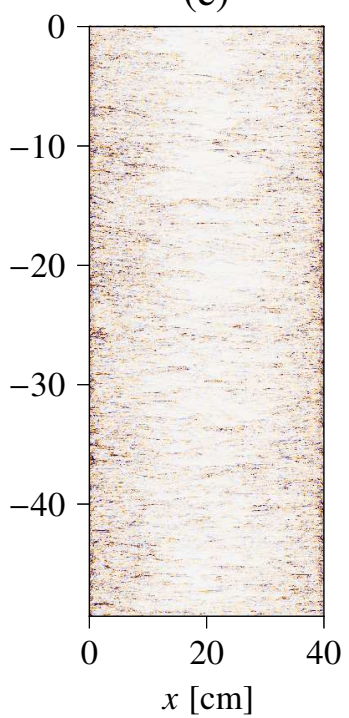

(c)

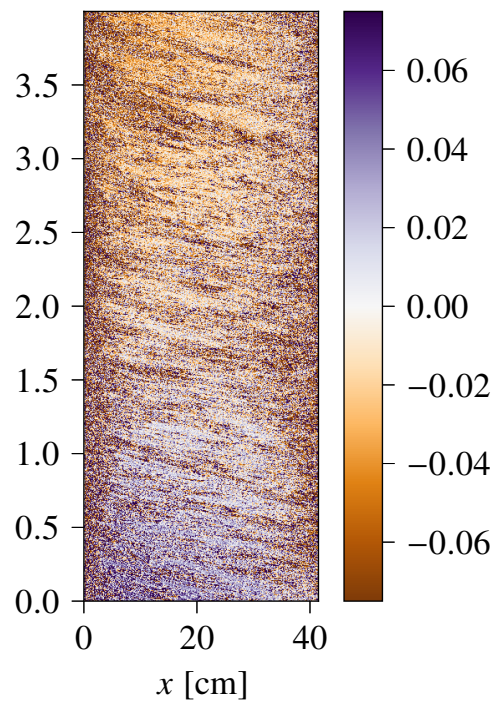

(f)

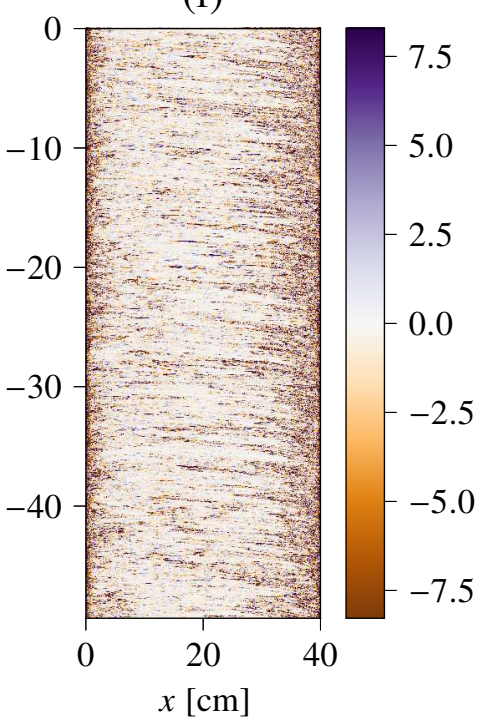

Figure 5: Spatio-temporal diagram of the shadowgraph intensity $I\left(x, z_{0}, t\right)$. Experimental diagrams at $R a=$ $5.6 \times 10^{10}$ : (a) $z_{0}=5 \mathrm{~cm}$ (bottom), (b) $z_{0}=20.75 \mathrm{~cm}$, (c) $z_{0}=36.5 \mathrm{~cm}$ (top). Numerical diagrams at $R a=2 \times 10^{9}$ : (d) $z_{0}=4.8 \mathrm{~cm}$ (bottom), (e) $z_{0}=20 \mathrm{~cm}$, (f) $z_{0}=35 \mathrm{~cm}$ (top). The $t$ axis of the numerical shadowgraph is reversed to visually match the experimental diagrams where the roll direction is opposite. 
(a)

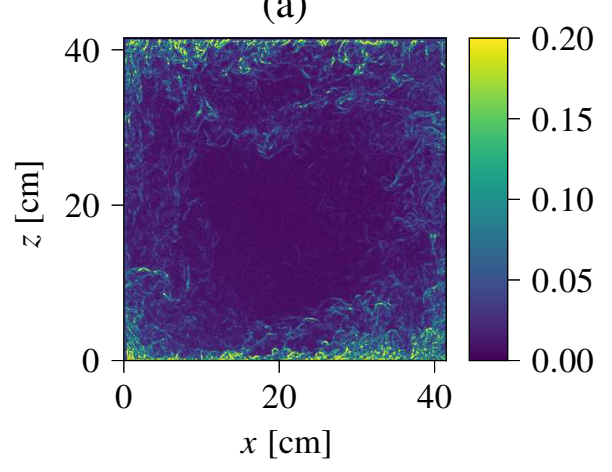

(c)

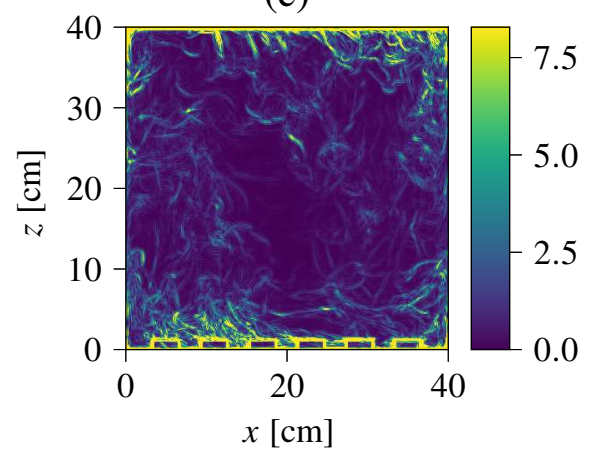

(b)

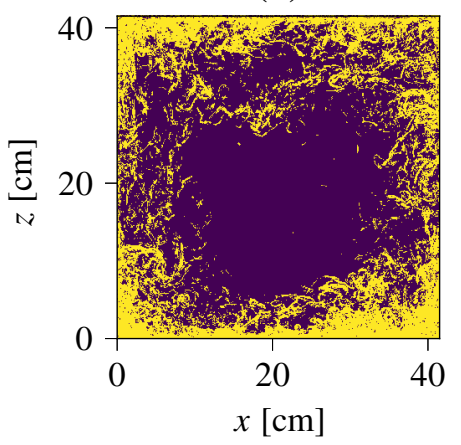

(d)

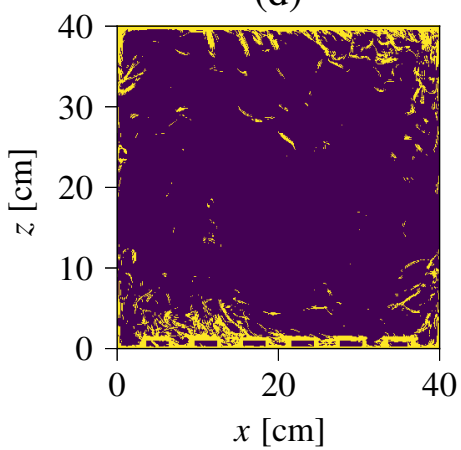

Figure 6: Local spatial variance of the shadowgraph images shown in figure 2, computed on a neighbourhood of $3 \times 3$ pixels, for the experimental image at $R a=5.6 \times 10^{10}$ (a), and for the numerical shadowgraph at $R a=2 \times 10^{9}$ (c). Texture segmentation obtained from thresholding the local variance, (b) for the experimental image with a threshold value 0.025 (in units of relative light intensity), and (d) for the numerical shadowgraph with a threshold value $3.1 \mathrm{~K} / \mathrm{cm}$ (in units of integrated temperature Laplacian). 
(a)

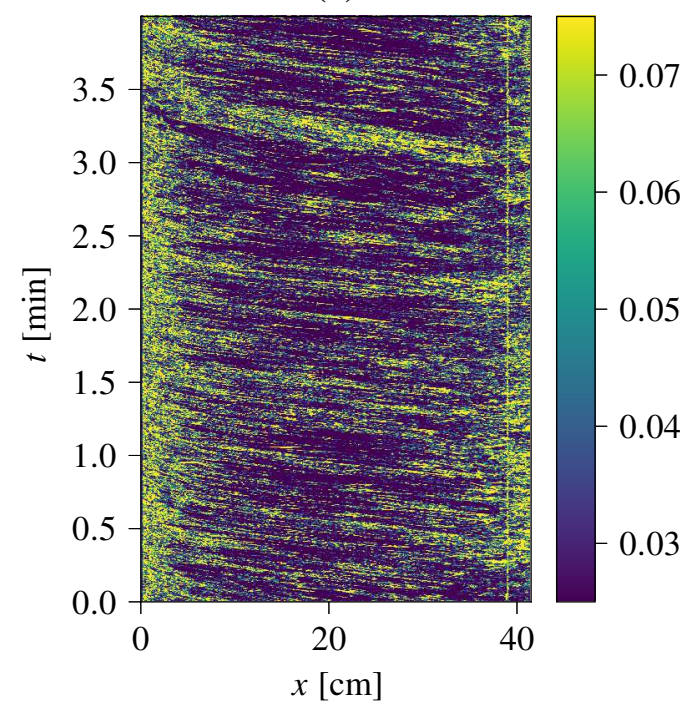

(c)

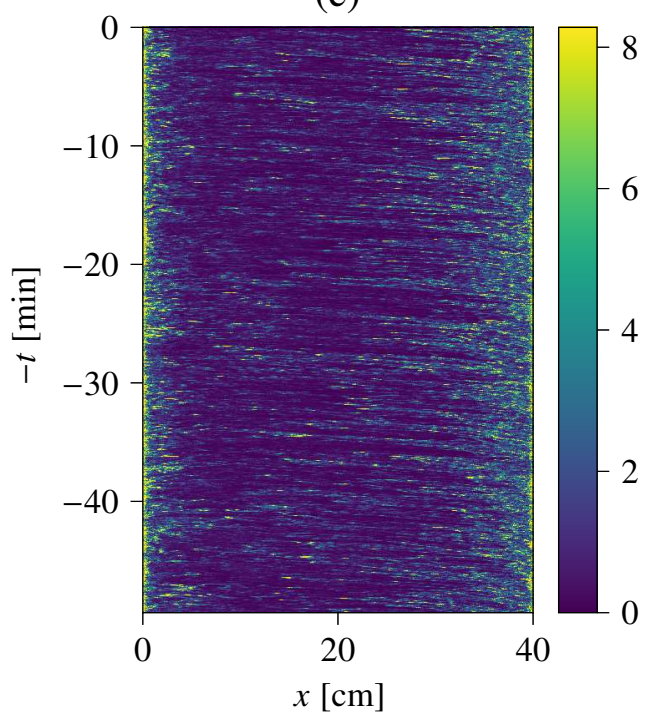

(b)

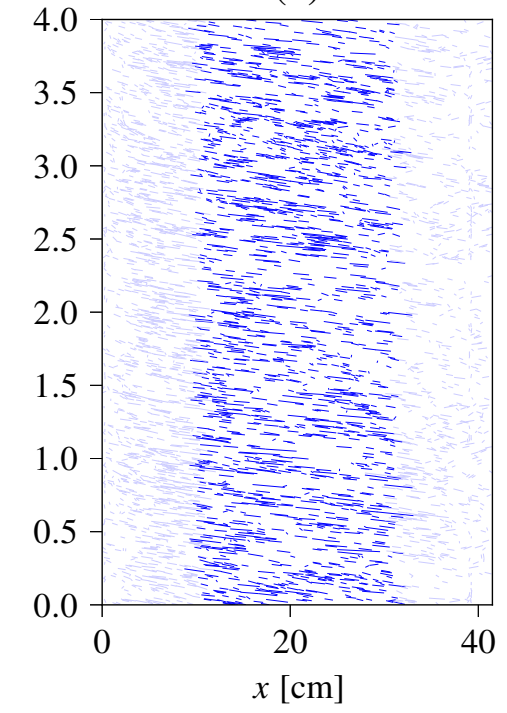

(d)

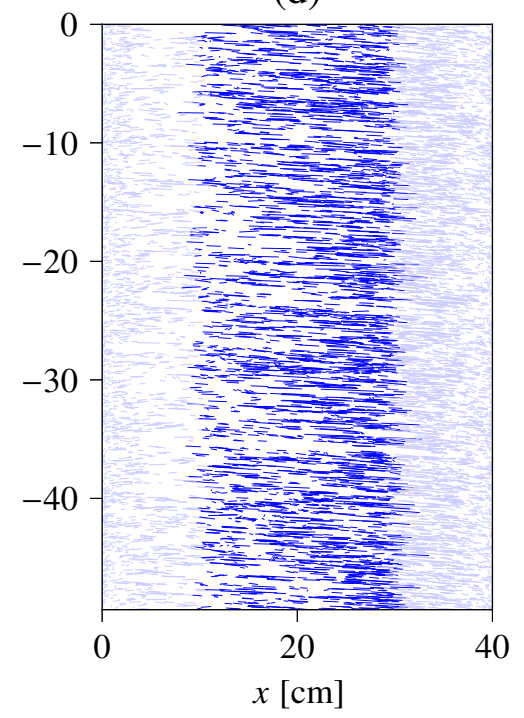

Figure 7: Spatio-temporal diagrams built from the horizontal line at $z_{0}$ of the local spatial variance shown in figure 6. (a) Experimental diagram at $R a=5.6 \times 10^{10}$ for $z_{0}=38.5 \mathrm{~cm}$ (top). (b) Reconstructed line segments from the experimental diagram, using OpenCV Line Segment Detector. (c) Numerical diagram at $R a=2 \times 10^{9}$ for $z_{0}=36 \mathrm{~cm}$ (top). The $t$ axis is reversed to visually match the experimental diagram, because the mean wind goes in the opposite direction. (d) Reconstructed line segments from the numerical diagram, using OpenCV Line Segment Detector. 
(a)

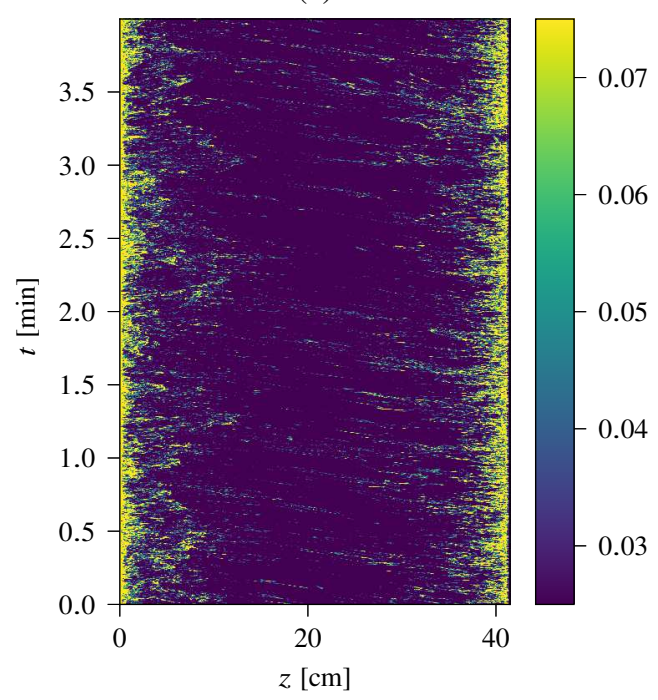

(c)

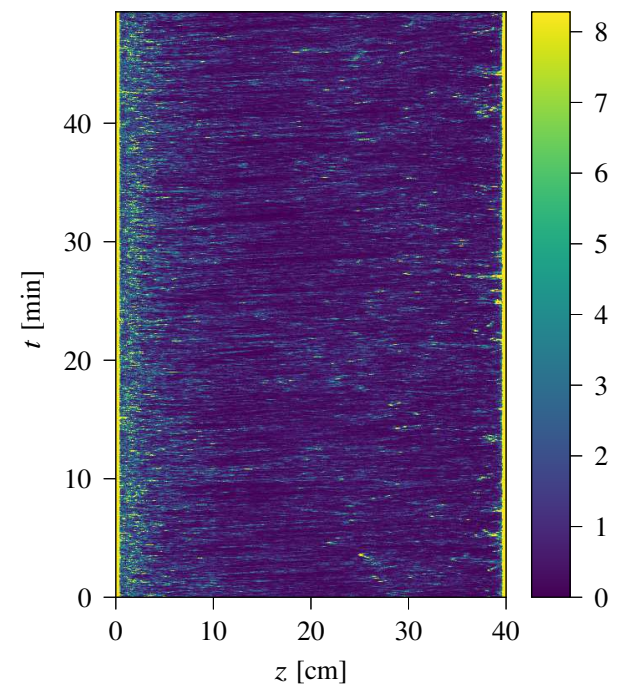

(b)

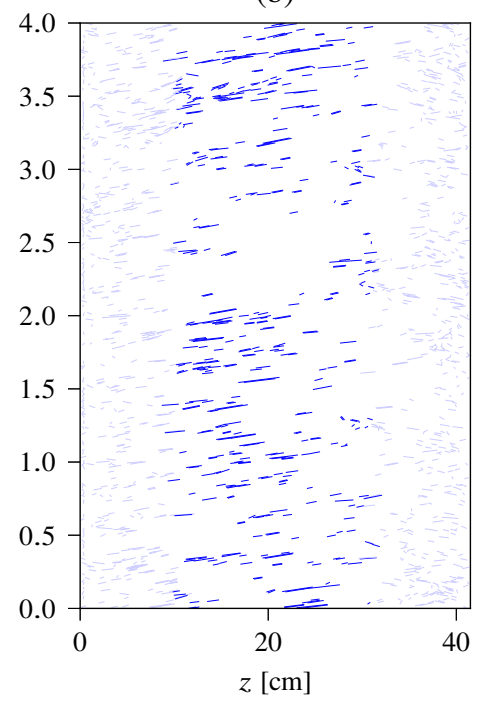

(d)

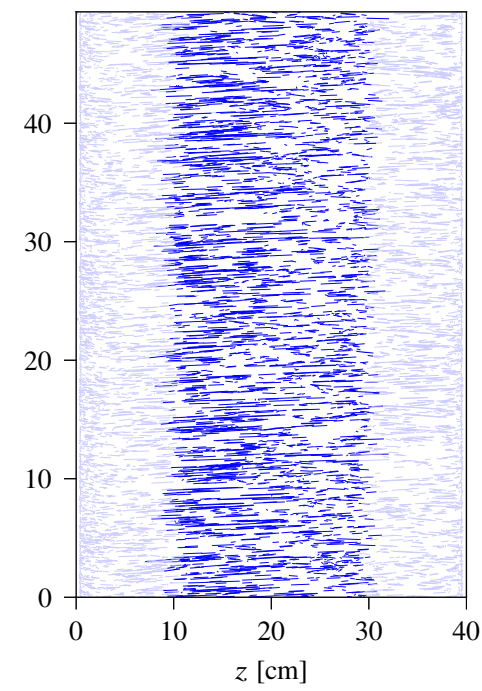

Figure 8: Spatio-temporal diagram built from the vertical line at $x_{0}=8 \mathrm{~cm}$ of the local spatial variance shown in figure 6. (a) Experimental diagram at $R a=5.6 \times 10^{10}$. (b) Reconstructed lines from the experimental diagram, using OpenCV Line Segment Detector. (c) Numerical diagram at $R a=2 \times 10^{9}$. (d) Reconstructed lines from the numerical diagram, using OpenCV Line Segment Detector. 


\subsection{Plume density}

Each detected segment corresponds to the advection of a thermal plume. However, because some segments are not detected, and because one segment could possibly originate from a group of plumes, the number of detected lines is not directly equal to the number of plumes. Nevertheless, the slope of the detected segments yields an estimate of the plume horizontal velocity, $U_{x}\left(x, z_{0}, t\right)$ for diagrams built from horizontal lines at $z=z_{0}$, or the plume vertical velocity $U_{z}\left(x_{0}, z, t\right)$ for diagrams built from vertical lines at $x=x_{0}$, and the number of detected lines, $N_{p l, h}$ and $N_{p l, v}$, are estimates of the plume density in both cases.

The detected segments in the case of a spatio-temporal diagram built from a vertical line, are shown in figure 8-b and $\mathrm{d}$. In this convention where the origin is at the bottom, we get positive vertical velocity for $z \rightarrow 0$, and negative vertical velocity for $z \rightarrow h$. This is a signature of rising plumes at the bottom, and falling plumes at the top. In the case of asymmetrical cells, where only the bottom plate is rough, this analysis allows to directly compare plume statistics of the smooth and rough plates, with no difference in the optical and image processing parameters.

The sets of line segments can be restricted those at the centre of the cell, $\xi<x<\ell-\xi$, and $\xi<z<h-\xi$, to obtain profiles similar to the kind of profiles found with a probe moving along the axis. We choose $\xi / h=1 / 4$ which is a compromise to have enough events to statistically converge the profile, and is sufficiently away from the walls to be considered nearly at the centre. The resulting number of plumes (per unit time) is shown in figure 9-a,b,c,d, for horizontally advected plumes $N_{p l, h}^{\text {center }}\left(z_{0}\right)$, and vertically advected plumes $N_{p l, v}^{\text {center }}\left(x_{0}\right)$.

It is also possible to count to total number of plumes, as a function of plate distance, $N_{p l}^{\text {tot }}(z)$. Indeed, for a vertical line at $x=x_{0}$, which yield vertically advected plumes at $x=x_{0}$, each detected line segment in the diagram has spatio-temporal coordinates $\left(z_{1}, t_{1}, z_{2}, t_{2}\right)$. One can build the histogram of the space coordinate of their centre positions, $N_{p l, v}\left(x_{0}, z\right)$. The total number of plumes $N_{p l}^{\text {tot }}$, shown in figure 9-e and f, is

$$
N_{p l}^{\mathrm{tot}}(z)=N_{p l, h}(z)+\sum_{x_{0}} N_{p l, v}\left(x_{0}, z\right)
$$

where $N_{p l, h}(z)$ is the number of advected plumes on the full horizontal line at $z$.

This measurement of the plume density can be compared with predictions and measurements in the literature. In their analysis of the role of plumes in thermal convection, Grossmann \& Lohse (2004) predicted that the number of plumes, $N_{p l}^{\text {tot }}$ scales like

$$
N_{p l} \sim N u .
$$

In our case, this means that the number of plumes in the experimental case $(N u=298.2)$ should be three times larger than the number of plumes in the simulation $(N u=107)$. As can be seen in figure 9 -e and $\mathrm{f}$, for $z / h \sim 0.2$, we find indeed around 5 plumes per second in the numerical simulation, and 15 plumes per second in the experiment.

One expected effect of plate roughness is to change the emission of thermal plumes. Du \& Tong (2000) found that the roughness elements on the plate enhance the detachment of the thermal boundary layer. Although the details of the cell geometry is different in our case, we also previously evidenced bursts of plume emissions near the rough plate, typically between $40 \delta_{t h}$ and $120 \delta_{t h}$ away from the plate, where

$$
\delta_{t h}=\frac{H}{2 N u}
$$

is the thermal boundary layer thickness (Salort et al., 2014).

In the experiment the thermal boundary layer is typically $\delta_{t h} \simeq 700 \mu \mathrm{m}$, more than twice smaller than the height of the roughness elements. In this regime, the plate is hydrodynamically rough, and the heat transfer is enhanced.

However, as can be seen in figure 9-a, the average number of detected plumes in the present experimental case on the upper and lower half of the cell only slightly differs. There are more plumes in the range $50 \delta_{t h}<z<150 \delta_{t h}$, which is where burst of plumes were previously observed. There are no visible difference far from the plates, when the number of detected plumes vanishes. There seems to be a difference very close to the plate in figure 9-a, with less detected plumes in the rough case. We do not know, at this stage, if our analysis holds so close to the plate, where spurious reflections are possible. We intend to operate similar experiment on a smooth symmetrical cell to compare. However, further away from the plate, no spurious optical effect is expected. Similar result is obtained from the number of rising and falling plumes in the jets (figure 9-c): the number of detected rising plumes is slightly higher than the number of detected falling plumes.

In the numerical simulation, the Rayleigh number is $R a=2 \times 10^{9}$, and the thermal boundary layer thickness is typically $\delta_{t h} \simeq 1.868 \mathrm{~mm}$, almost ten time smaller than the height of the roughness element. Compared to the experimental case, figure 9-b show that the plume statistics more strongly differ between the top and the bottom: 

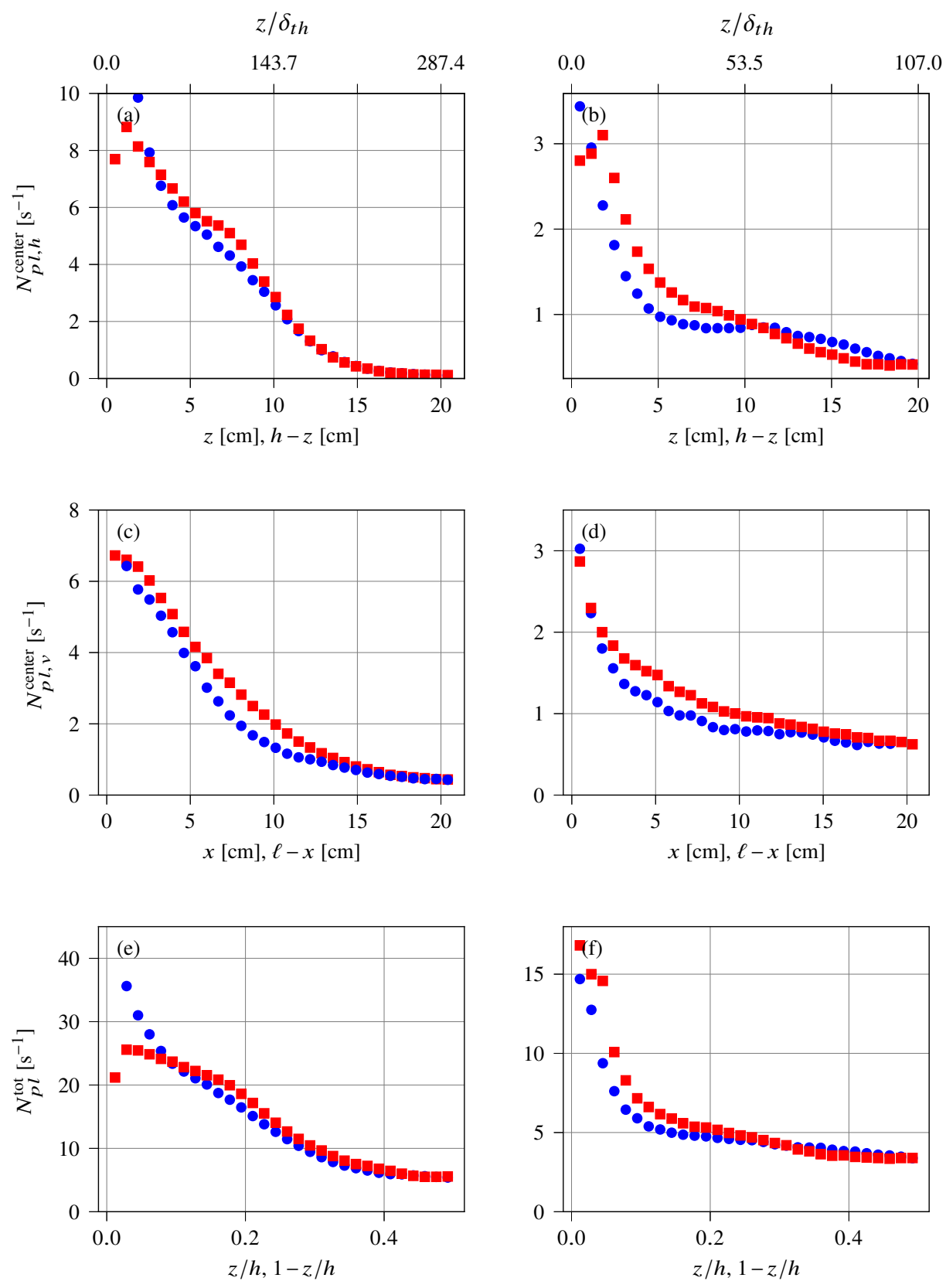

Figure 9: Number of detected segments on the spatio-temporal diagram, per unit time. (a) and (b): spatio-temporal diagram from horizontal lines, detecting plumes moving horizontally, as a function of the plate distance $z$, restricted to the centre area, $\xi<x<\ell-\xi$. Blue circles: upper smooth plate. Red squares: lower rough plate. (c) and (d): spatio-temporal diagram from vertical lines, detecting plumes moving vertically, as a function of the distance from the wall $x$, restricted to the centre area, $\xi<z<h-\xi$. Blue circles: falling plumes. Red squares: rising plumes. (e) and (f): Total number of plumes in the cell, at a given altitude $z$. (a), (c) and (e): Experiment at $R a=5.6 \times 10^{10}$. (b), (d) and (f): Numerical simulation at $R a=2 \times 10^{9}$. 
(a)

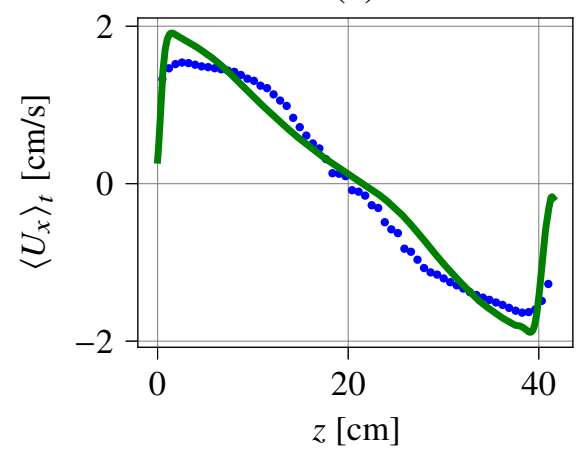

(c)

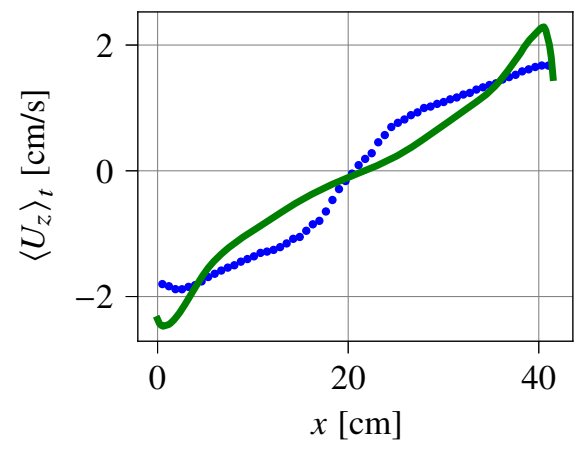

(b)

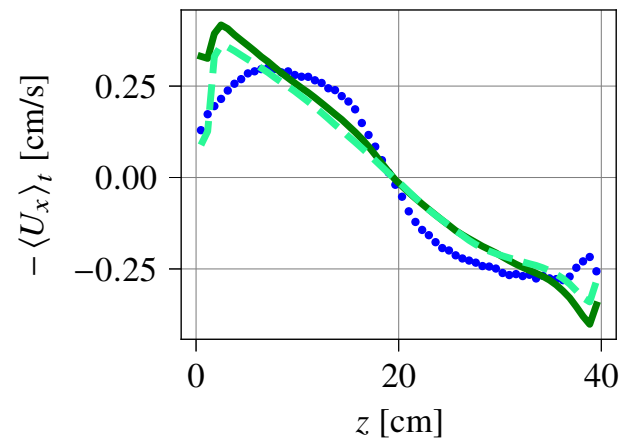

(d)

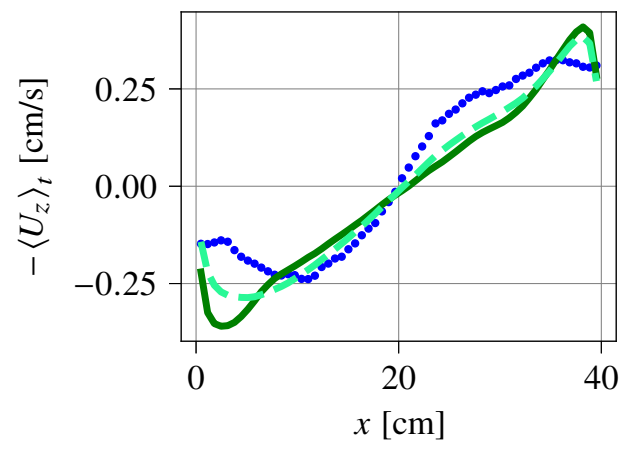

Figure 10: (a) and (c) Experimental horizontal (resp. vertical) velocity profile, obtained from the slope of the shadowgraph spatio-temporal diagram segments at $R a=5.6 \times 10^{10}$ (blue circles), or from PIV at $R a=6.2 \times 10^{10}$ (solid dark green line). (b) and (d) Numerical horizontal (resp. vertical) velocity profile, obtained from the slope of the numerical shadowgraph spatio-temporal diagram segments at $R a=2 \times 10^{9}$ (blue circles), from the mid-plane slice of the horizontal velocity average (solid dark green line), from the $y$-integrated horizontal velocity average (dashed light green line). The $\left\langle U_{x}\right\rangle$ and $\left\langle U_{z}\right\rangle$ axis of the simulation are reversed to visually match the experimental profile, where the roll direction is opposite. The average is both temporal and spatial for $\xi<x<\ell-\xi$ (resp. $\xi<z<h-\xi)$, and $\xi=\ell / 4$ (resp. $\xi=h / 4$ ).

there are more plumes near the bottom plate. Figure 9-d show also that there are more rising plumes than falling plumes.

The stronger plume enhancement observed in the numerical simulation may be a signature of the Regime III. Indeed, the experiment lies in Regime II at relatively high Rayleigh number, and the numerical simulation lies in Regime III, at lower Rayleigh number. In these conditions, the flow is more plume-dominated in the numerical simulations than in the experiment, which is more turbulence dominated. It is however a general feature that the rough plate yields more total plumes in both cases.

\subsection{Velocity statistics}

The analysis of the segments in the spatio-temporal diagram built from horizontal lines at $z=z_{0}$ yields sets of plume horizontal velocities, $U_{x}\left(x, z_{0}, t\right)$. From these sets, one can infer the average horizontal velocity $\left\langle U_{x}\right\rangle_{t, x}\left(z_{0}\right)$, as a function of the plate distance, see figure 10-a and b. The average is both temporal and spatial, but only lines near the centre $\xi<x<\ell-\xi$ are considered. In the experiment, these profiles can be compared to velocity profiles previously obtained from Particle Image Velocimetry (PIV) in similar conditions, and identical geometry. In the numerical simulation, we use the average velocities of the mid-plane, at $y=10 \mathrm{~cm}$, as a reference.

These velocity profiles can be compared to those found by Belmonte et al. (1994); Vasiliev et al. (2016) in a smooth Rayleigh-Bénard cell filled with water, where the velocity was measured with a $\mathrm{pH}$ dye technique. They found a maximum for both the velocity at $z / h \simeq 0.05$, i.e. at $z>\delta_{t h}$. Although our full cell images, either shadowgraph or PIV, do not have the resolution to accurately measure at the scale of boundary layer, similar maxima can be observed in our profiles, and at similar $z$. 
Similarly, the analysis of the segments of the spatio-temporal diagram built from the vertical lines at $x=x_{0}$ yields sets of plume vertical velocities, $U_{z}\left(x_{0}, z, t\right)$. From these sets, one can infer the average vertical plume velocity $\left\langle U_{z}\right\rangle_{t, z}\left(x_{0}\right)$, as a function of the distance from the wall, see figure 10-c and $\mathrm{d}$.

The plume velocity profiles, obtained from spatio-temporal diagrams or from fluid velocity measurement (PIV in experiment, mid-plane velocity in numerical simulation), have similar order of magnitude. It is is of order $2 \mathrm{~cm} / \mathrm{s}$ in the experiment, and $0.4 \mathrm{~cm} / \mathrm{s}$ in the numerical simulation. But the profiles do not completely collapse.

We use the numerical simulation to determine if this observed discrepancy is the consequence of the shadowgraph method, rather than an actual difference between plume velocities and fluid velocity. In principle, a difference between average plume velocity and average fluid velocity is not unphysical. Indeed, there is a selection bias: while the advection velocity of a plume should indeed be the same as the local velocity of the fluid, statistics based on plume advection measurements does not account for velocities of fluid parcels between plumes, or in areas where there is no plume. Additionally, the velocity field is divergence free, so a fluid parcel moving up (resp. down) must induce that there is fluid moving down (resp. up). This down-moving fluid parcel is not necessarily a falling plume, as it is moving down because of incompressibility and not because it is colder. The fluid velocity average accounts for all fluid movements, while the plume velocity statistics only accounts for the contribution of plumes.

Yet, the integration along the $y$ axis could lower the average, as fluid parcels slowed by the drag on the vertical walls will contribute in the integral. Integration may also smooth larger velocity events. Therefore, to check this hypothesis, we compute a profile of the $y$-averaged horizontal fluid velocity. This is similar to what we would get if PIV were integrated in the same manner as shadowgraph is. This profile is shown in light green dashed line in figure 10-b. As expected it is slightly lower than the profile obtained without integration in the $y$ direction. However, it does not differ from the non-integrated fluid velocity, as much as the plume velocity does. This indicates that the integration along $y$ is not responsible for the observed difference between plume velocity average and fluid velocity average.

It is very broadly assumed in the literature that the velocity of advected plumes can be a satisfactory estimate for the velocity of the "wind", which is then used to infer a Reynolds number. In practise, this is used mostly in cases where direct velocity measurement is not possible, using the correlation time between thermometers in the rising (or falling) jets (Wu, 1991; Chavanne et al., 2001; Sun \& Xia, 2005; He et al., 2015; Musilová et al., 2017), or correlation of shadowgraph images (Funfschilling \& Ahlers, 2004; Brown et al., 2007). While we find that the average plume velocity yields indeed the same order of magnitude as that of the mean fluid velocity, this analysis shows that care must be taken when comparing the Reynolds numbers computed in this fashion between different cells. He \& Tong (2011) have analysed the temperature correlation in homogeneous convection. Their result is in agreement with predictions from He \& Zhang (2006), and showed that estimates of velocity can be inferred. However, to interpret this velocity estimate properly, there is an underlying assumption that the plume velocity statistics and local velocity statistics are identical. Besides the present work, we are not aware of another direct comparison in the literature between the statistics of plume advection velocity versus local fluid velocity.

In particular, the velocity estimate from rising (or falling) plumes in the jets very close to the walls is close to the maximum fluid velocity, yet still lower. Further away from the wall, average plume velocities are found higher than the average fluid velocity. Assuming the velocity profiles shown in figure 10-c bear some universality, let us discuss the consequence for those results in the literature. They are all performed in cylindrical cells. Let us note $\Phi$ their diameter, and $r$ the distance from the wall. Wu (1991) and Musilová et al. (2017) have thermistors at a lateral position $r / \Phi=0.05$, He et al. (2015) have thermistors at a lateral position $r / \Phi \sim 0.013$. For those, the plume velocity may be $40 \%$ smaller than the fluid velocity, at least in this range of Rayleigh numbers. Chavanne et al. (2001) have thermistors at a lateral position $r / \Phi=0.25$, where plume velocity is slightly larger than average fluid velocity, but the fluid velocity is more than twice smaller than the velocity closer to the walls.

Finally, the velocity probability density function (PDF) are shown in figure 11. The PDF of the experimental and numerical data are mostly similar.

The velocity PDF, obtained from fluid velocity (PIV measurements in the experiment, mid-plane velocity in the numerical simulation), and those obtained from shadowgraph spatio-temporal diagram, are similar at $z=5 \mathrm{~cm}$ and $z=35 \mathrm{~cm}$. However, they seem to evidence exponential tails which may seem in contradiction with the data from Qiu et al. (2004) in a cylindrical cell, or Belmonte et al. (1994) in a cubic cell, where Gaussian velocity PDF are measured at the centre of the convection cell. But Zhou et al. (2007b) report exponential velocity PDF, away from the side walls in a rectangular cell, with a geometry much closer to ours. The difference between cylindrical and cubic, versus rectangular may arise from the contribution of azimuthal motion which is reported to have a Gaussian amplitude PDF (Xi et al., 2006).

In the experiment, small difference in the operating conditions (see table 1) could account for the slight differences. Some velocity PDF obtained from PIV exhibit secondary peaks (clearly visible for example in figure 11-c, 
(a)

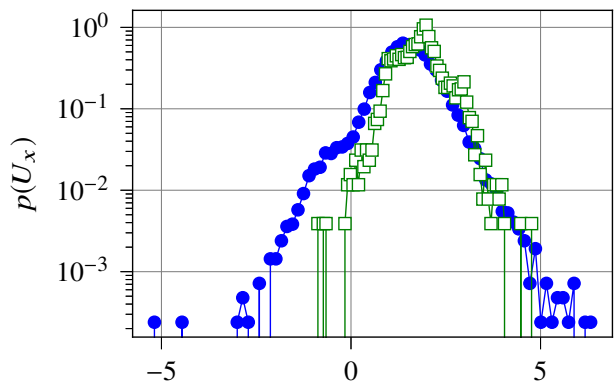

(b)

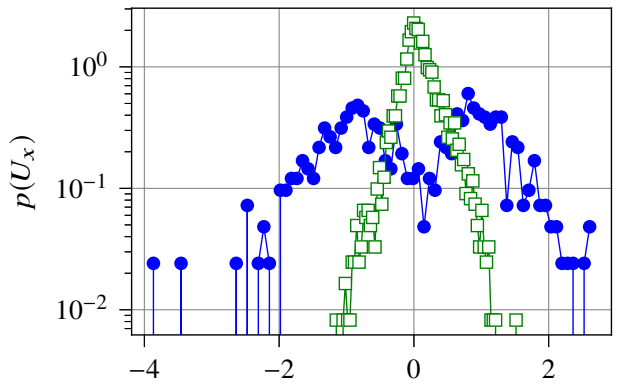

(c)

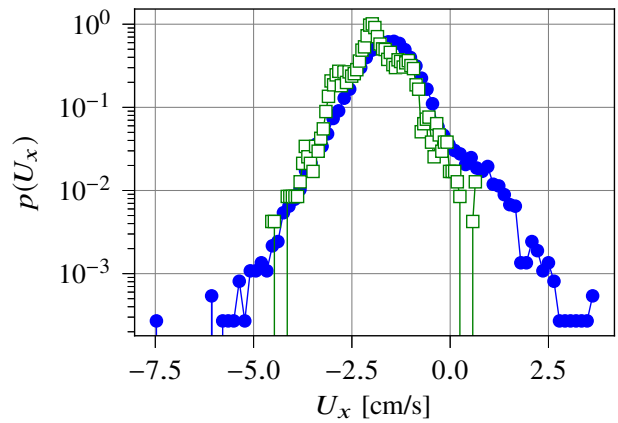

(d)

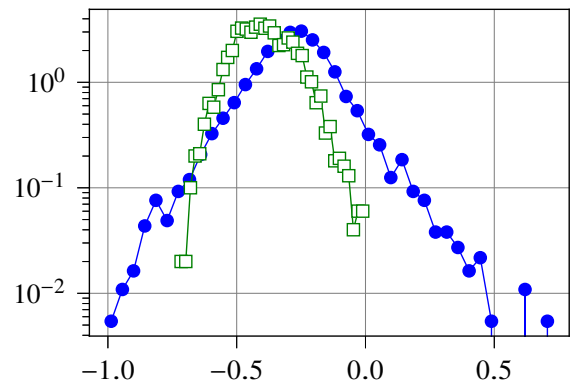

(e)

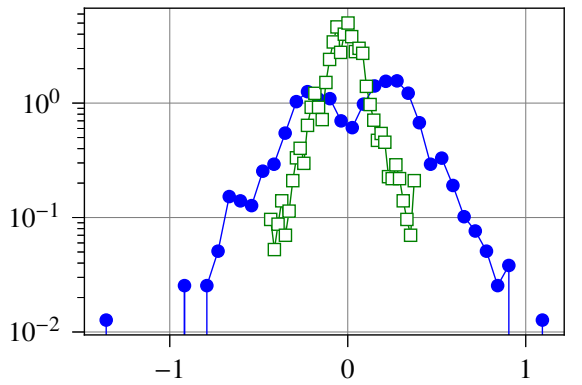

(f)

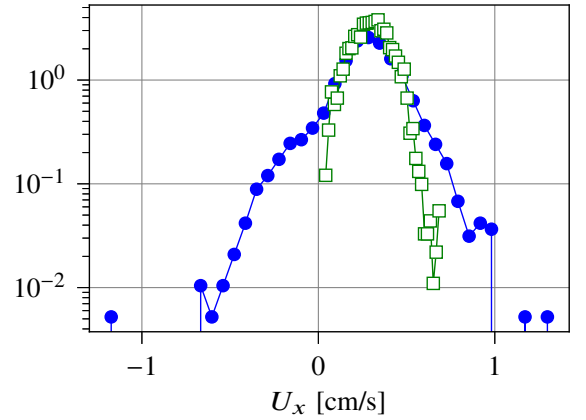

Figure 11: Velocity PDF computed from the shadowgraph spatio-temporal diagram lines (full circles), or from PIV measurements for the experimental data, or the horizontal velocity mid-plane slice for the numerical simulation (open squares). Experimental data at $R a=5.6 \times 10^{10}$ : (a) $5 \mathrm{~cm}$ above the bottom plate. (b) mid-height. (c) $5 \mathrm{~cm}$ below the top plate. Numerical simulations at $R a=2 \times 10^{9}$ : (d) $5 \mathrm{~cm}$ above the bottom plate. (e) mid-height. (f) $5 \mathrm{~cm}$ below the top plate. 
at $z \approx-2 \mathrm{~cm} / \mathrm{s}$ and $z \approx-3 \mathrm{~cm} / \mathrm{s}$ ). These velocities are close to integer pixel displacements and are due to peak locking, which are known artefacts of the sub-pixel estimator used by the PIV algorithm Raffel et al. (1998).

The PDF are however very different at the centre of the cell, where the fluid velocity exhibits a symmetric histogram centred on zero, while plume velocity PDFs evidence two peaks. The reason is that our method can only capture advected plumes, in one direction or another, but cannot resolve vanishing velocities, where no plume is advected.

The plume velocity histograms always evidence events in both directions $\left(U_{x}>0\right.$ and $\left.U_{x}<0\right)$, but the most probable plume velocity value matches the most probable fluid velocity. The plume velocity distributions at $z=5 \mathrm{~cm}$ and $z=35 \mathrm{~cm}$ are slightly skewed, which may be a signature of the two kinds of plumes that are captured: plumes emitted from the top plate and those emitted from the bottom plate, which each have their PDF. The final PDF is the superposition of the PDF of these two kinds of plumes.

\section{Conclusions}

We have shown that synthetic shadowgraph images can be computed in numerical simulations from the full temperature field, and that they are strikingly similar to experimental shadowgraph images. This confirms that the simple geometrical optics is a sufficient model for shadowgraphy in Rayleigh-Bénard convection. The contrast of the shadowgraph image can be greatly enhanced by computing the local variance of the image. Though there is no straightforward analytical relation, the DNS images show that this local variance shadowgraph image closely resembles the local thermal dissipation rate.

The local variance shadowgraph allows to build high contrast spatio-temporal diagrams, and opens the way to automatic detection of line segments. Statistics of thermal plumes can be inferred from these sets of line segments. The velocity estimated from the slope of these segments, and interpreted as plume velocity, has the same order of magnitude as the fluid mean velocity, but are not identical, with a relative difference up to $40 \%$. This is important because advected plume time-of-flight are widely used as estimates for the "wind" velocity, when direct velocity measurement is not possible. Additionally, other statistics such as the plume velocity PDF, may differ from fluid velocity $\mathrm{PDF}$, so one must exert caution when inferring higher order statistics.

The analysis of the plume density, in Rayleigh-Bénard cells with a rough bottom plate, and a smooth top plate, shows direct evidence of the increase of plume emission, as well as possible signatures of the transition between roughness-triggered plume dominated regime and roughness-triggered turbulent regime. In this asymmetric configuration, the number of rising hot plumes is larger than the number of falling cold plumes.

\section{ACKNOWLEDGEMENTS}

This numerical work was granted access to the HPC resources of GENCI-IDRIS under allocation 2a0326 made by GENCI. The experimental work benefited from funding by ANR-18-CE30-0007-01 JCJC "CryoGrad" project. The experiments could not have been possible without the help of Marc Moulin and the mechanical workshop who designed and built the experimental cell. The collaborative work between Limsi and LPENSL was funded by the CNRS INSIS PEPS Energie 2018 "CORINThe" joint project. Image processing benefited from the resources of the PSMN computing centre in Lyon.

The authors are grateful to Barbara Pascal and Nelly Pustelnik for an interesting discussion about image segmentation, and for suggesting to compute the local variance. We thank Bernard Castaing, David Dumont and Lucas Méthivier for proofreading of this manuscript.

Declaration of Interests. The authors report no conflict of interest.

\section{References}

Ahlers, G., Bodenschatz, E., Funfschilling, D., Grossmann, S., He, X., Lohse, D., Stevens, R. J. A. M. \& Verzicco, R. 2012 Logarithmic temperature profiles in turbulent Rayleigh-Bénard convection. Phys. Rev. Lett. 109, 114501.

Ahlers, G., Bodenschatz, E. \& He, X. 2017 Ultimate-state transition of turbulent Rayleigh-Bénard convection. Phys. Rev. Fluids 2, 054603.

Ahlers, G., Grossmann, S. \& Lohse, D. 2009 Heat transfer and large scale dynamics in turbulent RayleighBénard convection. Rev. Mod. Phys. 81, 503. 
BeLKADI, M. 2019 Numerical modeling of turbulent convection in rough Rayleigh-Bénard cell. PhD thesis.

Belmonte, A., Tilgner, A. \& Libchaber, A. 1994 Temperature and velocity boundary layers in turbulent convection. Phys. Rev. E 50 (1), 269-279.

Bouillaut, V., Lepot, S., Aumaître, S. \& Gallet, B. 2019 Transition to the ultimate regime in a radiatively driven convection experiment. J. Fluid Mech. 861 (R5), 1.

Bradski, G. 2000 The OpenCV Library. Dr. Dobb's Journal of Software Tools .

Brown, E., Funfschilling, D. \& Ahlers, G. 2007 Anomalous Reynolds-number scaling in turbulent RayleighBénard convection. J. Stat. Mech. p. P10005.

de Bruyn, J. R., Bodenschatz, E., Morris, S. W., Trainoff, S. P., Hu, Y., Cannel, D. S. \& Ahlers, G. 1996 Apparatus for the study of Rayleigh-Bénard convection in gases under pressure. Rev. Sci. Instrum. 67 (6), 2043.

Castaing, B., Gunaratne, G., Heslot, F., Kadanoff, L., Libchaber, A., Thomae, S., Wu, X., Zaleski, S. \& Zanetti, G. 1989 Scaling of hard thermal turbulence in Rayleigh-Bénard convection. J. Fluid Mech. 204, $1-30$.

Chavanne, X., Chillà, F., Castaing, B., Hébral, B., Chabaud, B. \& Chaussy, J. 1997 Observation of the ultimate regime in Rayleigh-Bénard convection. Phys. Rev. Lett. 79, 3648-3651.

Chavanne, X., Chillà, F., Chabaud, B., Castaing, B. \& Hébral, B. 2001 Turbulent Rayleigh-Bénard convection in gaseous and liquid He. Phys. fluids 13 (5), 1300-1320.

Chillà, F., Ciliberto, S., Innocenti, C. \& Pampaloni, E. 1993 Boundary Layer and Scaling Properties in Turbulent Thermal Convection. Il Nuovo Cimento D 15 (9), 1229.

Chillà, F., Rastello, M., Chaumat, S. \& Castaing, B. 2004 Ultimate regime in Rayleigh-Bénard convection: The role of plates. Physics of Fluids 16 (7), 2452-2456.

Chillà, F. \& Schumacher, J. 2012 New perspectives in turbulent Rayleigh-Bénard convection. Eur. Phys. J. E $35,58$.

Ciliberto, S., Francini, F. \& Simonelli, F. 1985 Real time measurements of optical disuniformity fields. Opt. Commun. 54 (5), 251-256.

Ciliberto, S. \& Laroche, C. 1999 Random roughness of boundary increases the turbulent convection scaling exponent. Phys. Rev. Lett. 82 (20), 3998.

Dalziel, S. B., Hugues, G. O. \& Sutherland, B. R. 2000 Whole-field density measurements by 'synthetic schlieren'. Exp. Fluids 28, 322-335.

Du, Y.-B. \& Tong, P. 1998 Enhanced heat transport in turbulent convection over a rough surface. Phys. Rev. Lett. 81 (5), 987-990.

Du, Y.-B. \& Tong, P. 2000 Turbulent thermal convection in a cell with ordered rough boundaries. J. Fluid Mech. 407, 57-84.

Du, Y.-B. \& Tong, P. 2001 Temperature fluctuations in a convection cell with rough upper and lower surfaces. Phys. Rev. E 63 (4), 046303.

Flór, J.-B., Scolan, H. \& Gula, J. 2011 Frontal instabilities and waves in a differentially rotating fluid. $J$. Fluid Mech. 685, 532-542.

Funfschilling, Denis \& Ahlers, Guenter 2004 Plume Motion and Large-Scale Circulation in a Cylindrical Rayleigh-Bénard Cell. Physical Review Letters 92 (19), 194502.

Funfschilling, D., Bodenschatz, E. \& Ahlers, G. 2009 Search for the "Ultimate State" in turbulent RayleighBénard convection. Phys. Rev. Lett. 103, 014503. 
García, A., Solano, J. P., Vicente, P. G. \& Viedma, A. 2012 The influence of artificial roughness shape on heat transfer enhancement: corrugated tubes, dimpled tubes and wire coils. Appl. Therm. Eng. 35, 196-201.

Gauthier, F., Salort, J., Bourgeois, O., Garden, J.-L., Du Puits, R., Thess, A. \& Roche, P.-E. 2009 Transition on local temperature fluctuations in highly turbulent convection. EPL 87, 44006.

Goluskin, D. \& Doering, C. R. 2016 Bounds for convection between rough boundaries. J. Fluid Mech. 804, $370-386$.

Grompone von Gioi, R., Jakubowicz, J., Morel, J.-M. \& Randall, G. 2012 LSD: a Line Segment Detector. Image Processing On Line 2, 35-55.

Grossmann, S. \& Lohse, D. 2000 Scaling in thermal convection: a unifying theory. J. Fluid Mech. 407, 27-56.

Grossmann, S. \& Lohse, D. 2004 Fluctuations in turbulent Rayleigh-Bénard convection: The role of plumes. Physics of Fluids 16 (12), 4462-4472.

Grossmann, S. \& Lohse, D. 2011 Multiple scaling in the ultimate regime of thermal convection. Phys. Fluids 23, 045108.

Hattori, T., Bartos, N., Norris, S. E., Kirkpatrick, M. P. \& Armfield, S. W. 2013 Experimental and numerical investigation of unsteady behaviour in the near-field of pure thermal planar plumes. Exp. Thermal and Fluid Science 46, 139-150.

He, G.-W. \& Zhang, J.-B. 2006 Elliptic model for space-time correlations in turbulent shear flows. Phys. Rev. E 73, 055303(R).

He, X., Funfschilling, D., Bodenschatz, E. \& Ahlers, G. $2012 a$ Heat transport by turbulent RayleighBénard convection for $\operatorname{Pr} \simeq 0.8$ and $4 \times 10^{11}<R a<2 \times 10^{14}$ : ultimate-state transition for aspect ratio $\gamma=1.00$. New J. Phys. 14, 063030.

He, X., Funfschilling, D., Nobach, H., Bodenschatz, E. \& Ahlers, G. $2012 b$ Transition to the ultimate state of turbulent Rayleigh-Bénard convection. Phys. Rev. Lett. 108, 024502.

He, X., van Gils, D. P. M., Bodenschatz, E. \& Ahlers, G. 2015 Reynolds numbers and the elliptic approximation near the ultimate state of turbulent Rayleigh-Bénard convection. New J. Phys. 17, 063028.

He, X. \& Tong, P. 2011 Kraichnan's random sweeping hypothesis in homogeneous turbulent convection. Phys. Rev. E 83, 037302.

Jenkins, D. R. 1988 Interpretation of shadowgraph patterns in Rayleigh-Bénard convection. J. Fluid Mech. 190, 451-469.

Kraichnan, R. H. 1962 Turbulent thermal convection at arbitrary Prandtl number. Phys. Fluids 5 (11), 13741389.

Liot, O., Ehlinger, Q., Rusaoü̈n, E., Coudarchet, T., Salort, J. \& Chillà, F. 2017 Velocity fluctuations and boundary layer structure in a rough Rayleigh-Bénard cell filled with water. Phys. Rev. Fluids 2, 044605.

Liot, O., Gay, A., Salort, J., Bourgoin, M. \& Chillà, F. 2016 a Inhomogeneity and Lagrangian unsteadiness in turbulent thermal convection. Phys. Rev. Fluids 1, 064406.

Liot, O., Salort, J., Kaiser, R., Du Puits, R. \& Chillà, F. $2016 b$ Boundary layer structure in a rough Rayleigh-Bénard cell filled with air. J. Fluid Mech. 786, 275-293.

Lovegrove, A. F., Read, P. L. \& Richards, C. J. 2000 Generation of inertia-gravity waves in a baroclinically unstable fluid. Q. J. R. Meteorol. Soc. 126, 3233-3254.

MacDonald, M., Hutchins, N., Lohse, D. \& Chung, D. 2019 Heat transfer in rough-wall turbulent thermal convection in the ultimate regime. Phys. Rev. Fluids 4, 071501(R).

Marat, J.-P. 1780 Recherches physiques sur le feu. C.A. Jombert (Paris). 
Musilová, V., Králik, T., Mantia, M. La, Macek, M., Urban, P. \& Skrbek, L. 2017 Reynolds number scaling in cryogenic turbulent Rayleigh-Bénard convection in a cylindrical aspect ratio one cell. J. Fluid Mech. 832, 721-744.

Ni, R., HuAng, S.-D. \& XIA, K.-Q. 2012 Lagrandian acceleration measurements in convective thermal turbulence. J. Fluid Mech. 692, 395-419.

Niemela, J. J., Skrbek, L., Sreenivasan, K. R. \& Donnelly, R. J. 2000 Turbulent convection at very high Rayleigh numbers. Nature 404, 837-840.

Pascal, B., Pustelnik, N., Abry, P., Serres, M. \& Vidal, V. 2018 Joint estimation of local variance and local regularity for texture segmentation. application to multiphase flow characterization. In 2018 25th IEEE International Conference on Image Processing (ICIP), IEEE International Conference on Image Processing ICIP , pp. 2092-2096. Inst Elect \& Electron Engneers; IEEE Signal Processing Soc, IEEE.

Du Puits, R., Resagk, C., Tilgner, A., Busse, F.H. \& Thess, A. 2007 Structure of thermal boundary layers in turbulent Rayleigh-Bénard convection. J. Fluid Mech. 572, 231-254.

Qiu, X.-L., Shang, X.-D., Tong, P. \& XIA, K.-Q. 2004 Velocity oscillations in turbulent Rayleigh-Bénard convection. Physics of Fluids 16 (2), 412-423.

Qiu, X.-L. \& Tong, P. 2001 Large-scale velocity structures in turbulent thermal convection. Phys. Rev. E 64 (3), 036304 .

QIU, X.-L., Xia, K.-Q. \& Tong, P. 2005 Experimental study of velocity boundary layer near a rough conducting surface in turbulent natural convection. J. Turbul. 6 (30), 1.

RAFFEL, M. 2015 Background-oriented schlieren (BOS) techniques. Exp. Fluids 56, 60.

Raffel, M., Willert, C., Wereley, S. T. \& Kompenhans, J. 1998 Particle Image Velocimetry. Experimental fluid mechanics. Springer Berlin Heidelberg.

Roche, P.-E., Castaing, B., Chabaud, B. \& Hébral, B. 2001 Observation of the 1/2 power law in RayleighBénard convection. Phys. Rev. E 63, 045303(R) 1-4.

Roche, P.-E., Gauthier, F., Kaiser, R. \& Salort, J. 2010 On the triggering of the ultimate regime of convection. New J. Phys. 12, 085014.

Rusaouen, E., Liot, O., Salort, J., Castaing, B. \& Chillà, F. 2018 Thermal transfer in Rayleigh-Bénard cell with smooth or rough boundaries. J. Fluid Mech. 837, 443-460.

Sakakibara, J. \& Adrian, R. J. 2004 Measurement of temperature field of a Rayleigh-Bénard convection using two-color laser-induced fluorescence. Exp. Fluids 37, 331-340.

Salort, J., liot, O., Rusaouen, E., Seychelles, F., Tisserand, J.-C., Creyssels, M., Castaing, B. \& Chillà, F. 2014 Thermal boundary layer near roughnesses in turbulent Rayleigh-Bénard convection: flow structure and multistability. Phys. Fluids 26, 015112.

Settles, G. S. 2001 Schlieren and Shadowgraph Techniques: Visualizing Phenomena in Transparent Media. Experimental fluid mechanics. Springer, Berlin.

Shishrina, O., Stevens, R. J. A. M., Grossmann, S. \& Lohse, D. 2010 Boundary layer structure in turbulent thermal convection and its consequences for the required numerical resolution. New J. Phys. 12, 075022.

Shishkina, O. \& Wagner, C. 2011 Modelling the influence of wall roughness on heat transfer in thermal convection. J. Fluid Mech. 686, 568-582.

Shraiman, B. I. \& Siggia, E. D. 1990 Heat transport in high-Rayleigh-number convection. Phys. Rev. A 42 (6), $3650-3653$.

Stasiek, J. A. \& Kowalewski, T. A. 2002 Thermochromic liquid crystals applied for heat transfer research. Opto-Electronics Review 10 (1), 1-10. 
Sun, Chao \& Xia, Ke-Qing 2005 Scaling of the Reynolds number in turbulent thermal convection. Phys. Rev. E $72(6), 067302$.

Taberlet, N., Plihon, N., Auzémery, L., Sautel, J., Panel, G. \& Gibaud, T. 2018 Synthetic schlieren application to the visualization and characterization of air convection. Eur. J. Phys. 39, 035803.

Tilgner, Andreas, Belmonte, A. \& Libchaber, A. 1993 Temperature and velocity profiles of turbulent convection in water. Phys. Rev. E 47 (4), R2253-R2256.

Tisserand, J.-C., Creyssels, M., Gasteuil, Y., Pabiou, H., Gibert, M., Castaing, B. \& Chillà, F. 2011 Comparison between rough and smooth plates within the same Rayleigh-Bénard cell. Phys. Fluids 23, 015105.

Toppaladoddi, S., Succi, S. \& Wettlaufer, J. S. 2017 Roughness as a Route to the Ultimate Regime of Thermal Convection. Phys. Rev. Lett. 118, 074503.

Trainoff, S. P. \& Cannell, D. S. 2002 Physical optics treatment of the shadowgraph. Phys. Fluids 14 (4), 1340.

Tummers, M. J. \& Steunebrink, M. 2019 Effect of surface roughness on heat transfer in Rayleigh-Bénard convection. Int. J. Heat Mass Trans. 139, 1056-1064.

Urban, P., Hanzelka, P., Králik, T., Macek, M. \& Musilová, V. 2019 Elusive transition to the ultimate regime of turbulent Rayleigh-Bénard convection. Phys. Rev. E 99, 011101(R).

Urban, P., Hanzelka, P., Musilová, V., Králik, T., Mantia, M. La, Srnka, A. \& Skrbek, L. 2014 Heat transfer in cryogenic helium gas by turbulent Rayleigh-Bénard convection in a cylindrical cell of aspect ratio 1. New J. Phys. 16, 053042.

Vasiliev, A., Sukhanovskit, A., Frick, P., Budnikov, A., Fomichev, V., Bolshukhin, M. \& Romanov, R. 2016 High Rayleigh number convection in a cubic cell with adiabatic sidewalls. Int. J. Heat Mass Trans. 102, 201-212.

Wagner, S. \& Shishkina, O. 2015 Heat flux enhancement by regular surface roughness in turbulent thermal convection. J. Fluid Mech. 763, 109-135.

Wang, Y., Lai, P.-Y., Song, H. \& Tong, P. 2018 Mechanism of large-scale flow reversals in turbulent thermal convection. Sci. Adv. 4 (11), eeat7480.

Wei, P. \& Ahlens, G. 2014 Logarithmic temperature profiles in the bulk of turbulent Rayleigh-Bénard convection for a Prandtl number of 12.3. J. Fluid Mech. 758, 809-830.

Wei, P., Chan, T.-S., Ni, R., ZhaO, X.-Z. \& Xia, K.-Q. 2014 Heat transport properties of plates with smooth and rough surfaces in turbulent thermal convection. J. Fluid Mech. 740, 28-46.

Wu, X.-Z. 1991 Along a road to developed turbulence : free thermal convection in low temperature Helium gas. PhD thesis.

XI, H.-D., LAM, S. \& XIA, K.-Q. 2004 From laminar plumes to organized flows: the onset of large-scale circulation in turbulent thermal convection. Journal of Fluid Mechanics 503, 47.

Xi, Heng-Dong, Zhou, Quan \& Xia, Ke-Qing 2006 Azimuthal motion of the mean wind in turbulent thermal convection. Physical Review E (Statistical, Nonlinear, and Soft Matter Physics) 73 (5), 056312.

XiA, K.-Q., Sun, C. \& Zhou, S.-Q. 2003 Particle image velocimetry measurement of the velocity field in turbulent thermal convection. Phys. Rev. E 68 (6), 066303.

Xie, Y.-C., Cheng, B.-Y.-C., Hu, Y.-B. \& XiA, K.-Q. 2019 Universal fluctuations in the bulk of RayleighBénard turbulence. J. Fluid Mech. 878, R1.

XIE, Y.-C. \& XIA, K.-Q. 2017 Turbulent thermal convection over rough plates with varying roughness geometries. J. Fluid Mech. 825, 573-599.

Zhang, Y.-Z., Sun, C., BaO, Y. \& Zhou, Q. 2018 How surface roughness reduces heat transport for small roughness heights in turbulent Rayleigh-Bénard convection. J. Fluid Mech. 836 (R2). 
Zhou, Q., Li, C.-M., Lu, Z.-M. \& Liu, Y.-L. 2011 Experimental investigation of longitudinal space-time correlations of the velocity field in turbulent Rayleigh-Bénard convection. J. Fluid Mech. 683, 94-111.

Zhou, Quan, Sun, Chao \& Xia, Ke-Qing $2007 a$ Morphological Evolution of Thermal Plumes in Turbulent Rayleigh-Bénard Convection. Physical Review Letters 98 (7), 074501.

Zноu, Q. \& XiA, K.-Q. 2010 Physical and geometrical properties of thermal plumes in turbulent Rayleigh-Bénard convection. New J. Phys. 12, 075006.

Zhou, S.-Q., Sun, C. \& Xia, K.-Q. $2007 b$ Measured oscillations of the velocity and temperature fields in turbulent Rayleigh-Bénard convection in a rectangular cell. Phys. Rev. E 76, 036301.

Zhou, S.-Q., Xie, Y.-C., Sun, C. \& Xia, K.-Q. 2016 Statistical characterization of thermal plumes in turbulent thermal convection. Phys. Rev. Fluids 1, 054301.

Zhu, X., Mathai, V., Stevens, R. J. A. M., Verzicco, R. \& Lohse, D. 2018 a Transition to the Ultimate Regime in Two-Dimensional Rayleigh-Bénard Convection. Phys. Rev. Lett. 120, 144502.

Zhu, X., Stevens, R. J. A. M., Shishrina, O., Verzicco, R. \& Lohse, D. $2019 N u \sim R a^{1 / 2}$ scaling enabled by multiscale wall roughness in Rayleigh-Bénard turbulence. J. Fluid Mech. 869, R4.

Zhu, X., Stevens, R. J. A. M., Verzicco, R. \& Lohse, D. 2017 Roughness-Facilitated Local 1/2 Scaling Does Not Imply the Onset of the Ultimate Regime of Thermal Convection. Phys. Rev. Lett. 119, 154501.

Zhu, X., Verschoof, R. A., Bakhuis, D., Huisman, S. G., Verzicco, R., Sun, C. \& Lohse, D. $2018 b$ Wall roughness induces asymptotic ultimate turbulence. Nature Physics 14, 417-423. 